\title{
Study on the coupling effect of controllable parameters on the dynamic characteristics of vehicle shift process throughout full life cycle of clutch
}

\section{Dongyang Wang}

Chongqing University

Minghui Hu ( $\nabla$ hu_ming@cqu.edu.cn )

Chongqing University https://orcid.org/0000-0001-7551-5883

\section{Datong Qin}

Chongqing University

\section{Original Article}

Keywords: Wet clutch, Service life cycle, Shift process, Controllable parameters, Dynamic characteristic

Posted Date: July 14th, 2020

DOI: https://doi.org/10.21203/rs.3.rs-40981/v1

License: (c) (1) This work is licensed under a Creative Commons Attribution 4.0 International License. Read Full License

Version of Record: A version of this preprint was published at SAE International Journal of Vehicle Dynamics, Stability, and NVH on August 6th, 2021. See the published version at https://doi.org/10.4271/10-05-04-0033. 


\section{Title page}

\section{Study on the coupling effect of controllable parameters on the dynamic characteristics of vehicle shift process throughout full life cycle of clutch}

Dong-Yang Wang, born in 1990, is currently a PhD candidate at School of Automotive Engineering, Chongqing University, China. He received his bachelor degree from Henan University of Science and Technology, China, in 2014. His research interests include Vehicle driveline system and control.

E-mail: wdyang@cqu.edu.cn

Ming-Hui Hu, born in 1975, is currently a professor at Chongqing University, China. He received his Ph.D. degree on mechanical design in Chongqing University, China, in 2007.

Tel: +86-15826089252; E-mail: hu_ming@cqu.edu.cn

Da-Tong Qin, born in 1956, is currently a professor at State Key Laboratory of Mechanical Transmissions, Chongqing University, China.

E-mail: dtqin@cqu.edu.cn

Corresponding author: Ming-Hui Hu E-mail: hu_ming@cqu.edu.cn 


\title{
Study on the coupling effect of controllable parameters on the dynamic characteristics of vehicle shift process throughout full life cycle of clutch
}

\author{
Dong-Yang Wang ${ }^{1}$ Ming-Hui Hu, ${ }^{1,2^{*}} \cdot$ Da-Tong Qin ${ }^{1,2}$
}

Received June xx, 201x; revised February xx, 201x; accepted March xx, 201x

(C) Chinese Mechanical Engineering Society and Springer-Verlag Berlin Heidelberg 2017

\begin{abstract}
In this study, coupling effect of controllable parameters on the dynamic characteristics of vehicle shift process throughout full life cycle of wet clutch was studied. Firstly, an improved dynamic model of driveline was established with consideration of friction characteristics of clutch, time-varying mesh stiffness of gears, and torque-coupling effect. Then, dynamic characteristics of driveline during shift process were analyzed, and the model was validated with an experiment. Based on the model, the effect of frictional characteristics of clutch on the dynamic characteristics of driveline were analyzed, and to reduce the adverse effects caused by the degradation of frictional characteristics on the dynamic characteristics of driveline, a control strategy was proposed, which control the rising rate of on-coming clutch pressure and the maximum pressure coordinately. the influence of these two controllable parameters on the dynamic characteristics of driveline during shift process throughout the whole life of wet clutch life was analyzed. The results showed that side effect caused by the degradation of clutch frictional characteristics on the driveline during shift process can be attenuated by controlling the rising rate of on-coming clutch pressure and the maximum pressure coordinately.
\end{abstract}

Keywords: Wet clutch - Service life cycle - Shift process • Controllable parameters $\bullet$ Dynamic characteristic

\section{Introduction}

Ming-Hui Hu

hu_ming@cqu.edu.cn

1 School of Automotive Engineering, Chongqing University, Chongqing 400044, China

2 State Key Laboratory of Mechanical Transmissions, Chongqing University, Chongqing 400044, China
As the issues of fuel consumption and environmental protection are rapidly gaining attention worldwide, vehicle manufacturers have increasingly been adopting new technology to improve the efficiency of all vehicle components. The innovative dual clutch transmission (DCT) combines the advantages of manual transmission and automatic transmission, which gives it high efficiency and uninterrupted traction [1-6]. Since Volkswagen launched the first automatic transmission with a dry clutch in the Golf class at the beginning of 2008, a large number of drivers and automotive manufacture experts have used this technology. The system is earning high ratings in various publications and comparative analyses. It meets and even exceeds expectations in terms of driving characteristics and fuel consumption.

Structurally, the DCT is a merger of two manual transmissions. It employs two separate clutches, which hold different gears. One clutch holds odd gears and the other holds even gears. When shifting, the two clutches engage and disengage alternately owing to the slip of clutches, which guarantees shift without interrupting the traction from the engine. Considerable researches have been conducted on the DCT structure to better understand this sophisticated system and improve the quality of transmission [7]. To reduce the viscous torque in wet DCT and create a seamless gear preselect, Adhitya et al. proposed a new gear preselect strategy by delaying the gear preselect time near the power shift point based on a 13-degree-of-freedom lumped inertia driveline model [1]. Walker et al. modeled the traction routine of the transmission, studied the dynamic characteristics of the traction routine [8-10]. Goetz et al. proposed an integrated control for gearshift based on the dynamic model of DCT $[11,12]$. Kulkarni et al. designed an integrated vehicle 
model and analyzed the influence of pressure profile of actuation mechanism on the output torque during shift process [13]. To improve shift comfort, Yun et al. designed a nonlinear gearshift controller for DCT based on the model dynamic characteristics [14]. Jiwon et al. also proposed a torque estimation method for each clutch of the dual clutch system $[15,16]$. Walker et al. investigated the nonlinear characteristics of driveline with consideration of the characteristics of clutch and the mesh contacts of the gear set [17].

DCT consists of three key components: dual clutch, gearbox, and actuation mechanism modules. Currently, researchers are focusing on the dual clutch and actuation mechanism modules, and effect of external excitation such as engine torque fluctuation, hydraulic pressure fluctuation on the dynamic characteristics were analyzed with relatively simple model [18-20]. The dynamic characteristics of the gearbox are highly nonlinear and coupled since the friction coefficient of clutch is variant when the clutch is slipping, and degrades with the service life, also, the mesh force between gears are time-varying. All these internal excitations of components affect the dynamic characteristics of powertrain, especially in shift process, during which one clutch engages while the other disengages, and two gears on the transfer shafts drive the output shaft simultaneously. It is essential to investigate the nonlinear and coupled characteristics of the gearbox to control the shift process effectively.

In terms of clutch control, more researches focused on the dynamic characteristics of DCT during shift process and designed controllers based on the dynamic characteristics, which are obtained with relatively simple model [21-23]. Few research considers the variation of dynamics characteristics of components in the driveline.

The purpose of this study is to investigate the effect of frictional characteristics of clutch on the dynamic characteristics of shift process throughout the whole life and find proper control strategy to attenuate the adverse effect. Firstly, an improved model was established with consideration of the frictional characteristics of clutch, the time-varying mesh stiffness of gear set, and torque coupling effect. Then, the shift process was analyzed and the model was validated by a test. Finally, based on the model, the effect of frictional characteristics of clutch on the shift along with the service life was studied, and to reduce the adverse effects caused by the degradation of frictional characteristics on the dynamic characteristics of driveline, a control strategy was proposed, which control the rising rate of on-coming clutch pressure and the maximum pressure coordinately. the influence of these two controllable parameters on the dynamic characteristics of driveline during shift process at three different stages of wet clutch life was analyzed.

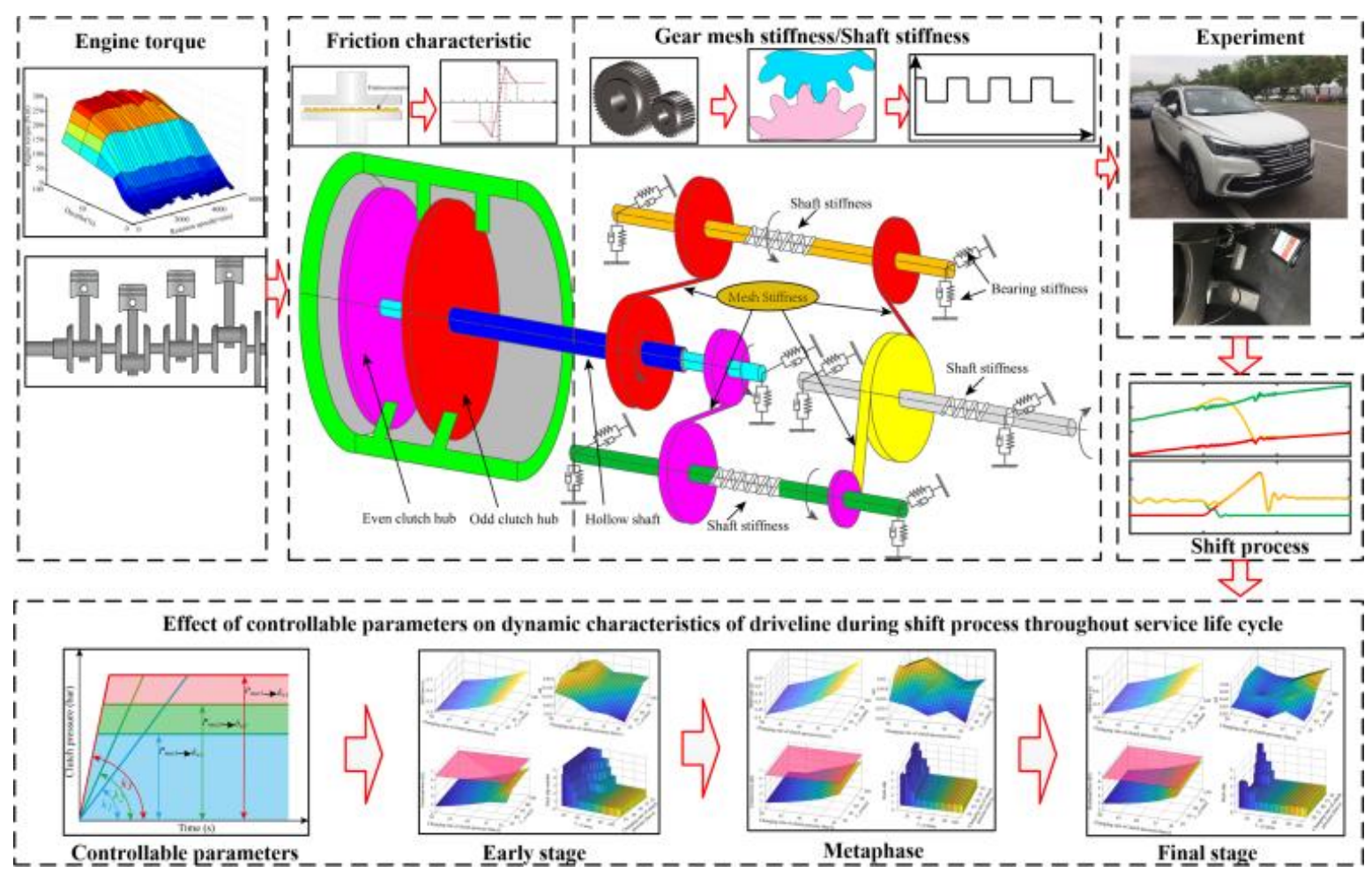

Figure 1 Structure of the paper 


\section{Modeling of DCT shift process}

\subsection{Engine model}

The internal combustion engine (ICE) is a complicated system, and, to analyze it thoroughly, multi-disciplinary knowledge of physics, chemistry, thermodynamics, fluid dynamics, mechanics, electronics, and process control is needed [19]. In this study, the torque generated by the ICE is a function of the opening degree of throttle and rotation speed, which can be expressed by equation (1), and the relationship can be illustrated by a map as can be seen in Figure 2.

$$
T_{e}=f\left(t h r t l, \omega_{e}\right)
$$

Where, $T_{e}$ is the output torque of engine, thrtl is the throttle of engine, and $\omega_{e}$ is the rotation speed of engine.

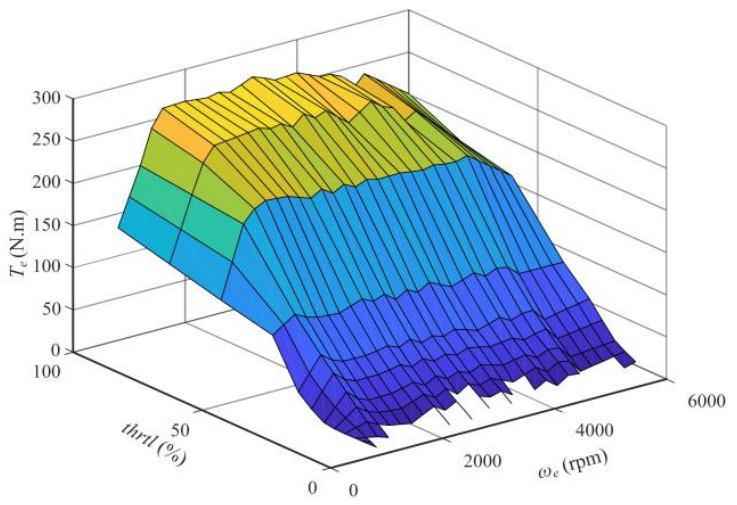

Figure 2 Map of the Engine

In this study, a DCT system, which has six forward speeds and one reverse speed, is shown in Figure 3. The DCT gearbox uses a coaxial structure to transfer power to the wheel. The solid shaft, which is installed inside the hollow shaft, carries the even gears and the hollow one carries the odd and reverse gears. Two transfer shafts carry the driven gears for each speed. In the gearbox, synchronizers exist between the two gears on the transfer shafts. This is similar to manual and automated manual transmission. When the gears are shifted, the synchronizer engages with the target gear, and the power from the engine flows through the clutch, synchronizer, gear set, final drive pinion, differential gear ring output shaft and wheel. In this section, models of key components in the gearbox were established, such as the clutch, the gears and the torque coupling device.

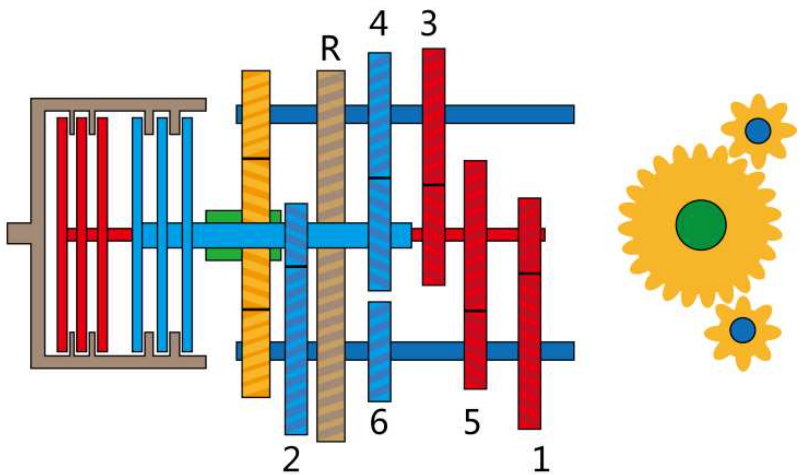

Figure 3 Schematic of DCT

\subsection{Clutch model}

The basic mechanism for torque generation in the clutch plate is the existence of a normal load and slip that together produce friction forces at distances from the plate center and result in a torque. Therefore, the amount of torque of clutch depends on the normal force and slip values. During engagement process, these parameters will vary with time and a transient torque transmission will result [24].

Generally, the working state of clutch can be divided into three states: separated state, slipping state, and locked state. When the clutch is in the separated state, no torque is transmitted, and this state is ignored in this research. In this study, the dynamic model of the clutch includes two states: slipping state and locked state. When working, the states of clutch switches between slipping and locked. In the driveline, the clutch works as a torque transition part connected with other components. The dynamic characteristic of clutch affects characteristics of the driveline, and that in return, other parts in the driveline will affect the engagement characteristics of clutch. To investigate the engagement characteristics of clutch, a 4 DOF (Degree of freedom) model is built, as shown in Figure 4.

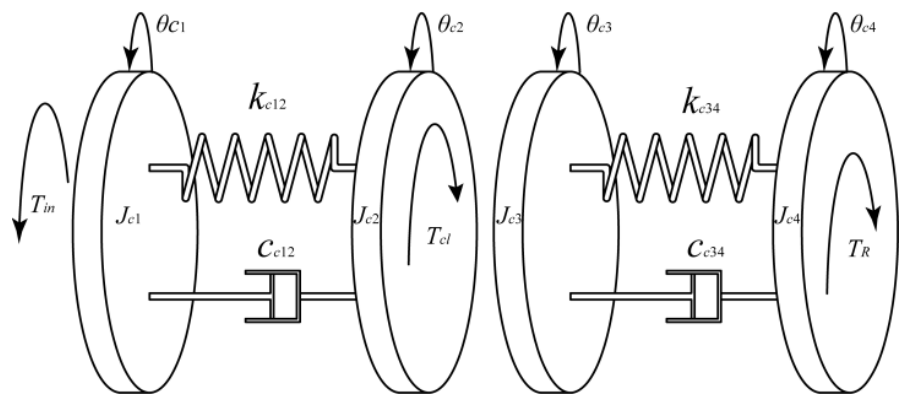

Figure 44 DOF model of the clutch

The mathematical model of clutch in slipping state can be expressed with equation (2-5). 


$$
\begin{aligned}
& J_{c 1} \ddot{\theta}_{c 1}=T_{i n}-k_{c 12}\left(\theta_{c 1}-\theta_{c 2}\right)-c_{c 12}\left(\dot{\theta}_{c 1}-\dot{\theta}_{c 2}\right) \\
& J_{c 2} \ddot{\theta_{c 2}}=k_{c 12}\left(\theta_{c 1}-\theta_{c 2}\right)+c_{c 12}\left(\dot{\theta}_{c 1}-\dot{\theta_{c 2}}\right)-T_{c l} \\
& J_{c 3} \ddot{\theta_{c 3}}=T_{c l}-k_{c 34}\left(\theta_{c 3}-\theta_{c 4}\right)-c_{c 34}\left(\dot{\theta_{c 3}}-\dot{\theta_{c 4}}\right) \\
& J_{c 4} \ddot{\theta_{c 4}}=k_{c 34}\left(\theta_{c 3}-\theta_{c 4}\right)+c_{c 34}\left(\dot{\theta_{c 3}}-\dot{\theta_{c 4}}\right)-T_{R}
\end{aligned}
$$

The mathematical model of clutch in locked can be expressed with equation (6-8).

$$
\begin{aligned}
& J_{c 1} \ddot{\theta}_{c 1}=T_{i n}-k_{c 12}\left(\theta_{c 1}-\theta_{l o c k}\right)-c_{c 12}\left(\dot{\theta_{c 1}}-\dot{\theta_{l o c k}}\right) \\
& \left(J_{c 2}+J_{c 3}\right) \theta_{l o c k}=k_{c 12}\left(\theta_{c 1}-\theta_{l o c k}\right)+c_{c 12}\left(\dot{\theta_{c 1}}-\dot{\theta}_{\text {lock }}\right) \\
& -k_{c 34}\left(\theta_{l o c k}-\theta_{c 4}\right)-c_{c 34}\left(\dot{\theta_{l o c k}}-\dot{\theta_{c 4}}\right) \\
& J_{c 4} \ddot{\theta}_{4}=k_{c 34}\left(\theta_{l o c k}-\theta_{c 4}\right)+c_{c 34}\left(\theta_{l o c k}-\dot{\theta_{c 4}}\right)-T_{R}
\end{aligned}
$$

Where, $\dot{\theta_{c 2}}=\dot{\theta}_{c 3}=\theta_{\text {lock }}$, when the clutch is locked. $T_{c l}$ is the torque transmitted by the clutch, which can be calculated by equation (9).

$$
\begin{aligned}
T_{c l} & =\mu\left(\omega_{s}\right) N \iint_{A} \frac{r z F}{A} d a=\frac{\mu\left(\omega_{s}\right) z F}{\pi\left(R_{o}^{2}-R_{i}^{2}\right)} \int_{R_{i}}^{R_{o}} \int_{0}^{2 \pi} r^{2} d r d \theta \\
& =2 / 3\left[\left(R_{o}^{3}-R_{i}^{3}\right) /\left(R_{o}^{2}-R_{i}^{2}\right)\right] \mu\left(\omega_{s}\right) z F \\
& =\mu\left(\omega_{s}\right) R z F
\end{aligned}
$$

Where, $R$ is the equivalent radius, $R=2\left(R_{o}{ }^{3}-R_{i}{ }^{3}\right) / 3 /\left(R_{o}{ }^{2}-R_{i}{ }^{2}\right), R_{o}$ is the outer radius of clutch lining and $R_{i}$ is the inner radius of clutch lining. $z$ is the number of contact faces. $F$ is the clamp force, $F=P \pi$ $\left(r_{p o}{ }^{2}-r_{p i}{ }^{2}\right) . P$ is the hydraulic pressure, $r_{p o}$ and $r_{p i}$ are the outer and inner radii of hydraulic cylinder, respectively. $\mu\left(\omega_{s}\right)$ is the friction coefficient, which is a function of relative sliding speed between clutch drum and hub, $\omega_{s} \cdot[25-26]$

$$
\mu\left(\omega_{s}\right)=\mu_{k}+\left(\mu_{s}-\mu_{k}\right) \exp \left(-\alpha \cdot \omega_{s} \cdot R^{\prime} \cdot \operatorname{sgn}\left(\omega_{s} \cdot R^{\prime}\right)\right)
$$

Where, $\alpha$ is the coefficient, which can be obtained by experiment, $\operatorname{sgn}()$ is the sign function, $\omega_{s}$ is the relative rotation speed, $R^{\prime}$ is the equivalent radius, $R^{\prime}=$ $3\left(R_{o}^{4}-R_{i}^{4}\right) / 4 /\left(R_{o}^{3}-R_{i}^{3}\right), u_{s}$ is the static friction coefficient, $u_{k}$ is the minimum dynamic friction coefficient.

The relationship between friction coefficient and relative rotation speed can be expressed with Taylor series, ignoring the high order items. $\mu\left(\omega_{s}\right)=\mu_{s}+k \omega_{s} R^{\prime}$

Where, $k$ is the coefficient, which is often referred to as negative gradient of friction coefficient. And, the torque transmitted by clutch in slipping and locked state can be calculated by equation (12)-(13).

$$
\begin{aligned}
& T_{c l}=\mu\left(\omega_{s}\right) \pi P\left(r_{p o}^{2}-r_{p i}^{2}\right) z R \\
& T_{\text {lock }}=\left\{\begin{array}{l}
J_{c 3}\left[k_{c 12}\left(\theta_{c 1}-\theta_{\text {lock }}\right)+c_{c 12}\left(\dot{\theta_{c 1}}-\theta_{\text {lock }}\right)\right] \\
+J_{c 2}\left[k_{c 34}\left(\theta_{\text {lock }}-\theta_{c 4}\right)+c_{c 34}\left(\dot{\theta_{\text {lock }}}-\dot{\theta_{c 4}}\right)\right]
\end{array}\right\} /\left(J_{c 2}+J_{c 3}\right)
\end{aligned}
$$

The torque transmitted by the clutch at different states can be expressed by equation (13).

$$
T_{f}= \begin{cases}\operatorname{sgn}\left(\dot{\theta_{c 2}}-\dot{\theta_{c 3}}\right) T_{c l} & \left|\dot{\theta}_{c 2}-\dot{\theta}_{c 3}\right| \geq \Delta \omega \\ \operatorname{sgn}\left(T_{\text {lock }}\right) T_{c l} & \left|\dot{\theta}_{c 2}-\dot{\theta}_{c 3}\right|<\Delta \omega, \quad\left|T_{\text {lock }}\right|>T_{\text {hold }} \\ T_{\text {lock }} & \left|\dot{\theta}_{c 2}-\dot{\theta_{c 3}}\right|<\Delta \omega, \quad\left|T_{\text {lock }}\right| \leq T_{\text {hold }}\end{cases}
$$

Where, $T_{\text {lock }}$ is the torque transmitted by the clutch in locked state, $T_{c l}$ is the torque transmitted by clutch in slipping state, $T_{\text {hold }}$ is the torque capacity of clutch, $T_{\text {hold }}=$ $\mu_{s} F z R, \Delta \omega$ is the tolerance of the relative slipping speed.

\subsubsection{Clutch engagement simulation result}

In this study, the Matlab/Simulink was used to obtain the numerical solution of the ODEs (Ordinary differential equations). Figure 5 shows the simulation result of clutch dynamic engagement process.

The upper graph in figure 5 shows the rotation speed of clutch drum and hub. During the engagement process, the rotation speed of clutch hub suffers oscillations and the amplitude of oscillation increases as the clutch engages, an action that is referred to as self-excited oscillation and is mainly caused by the negative gradient of friction coefficient [27]. Lower graph in figure 5 shows corresponding torque of clutch and relative ration speed of clutch drum and hub. In this figure, the frictional torque of clutch increases with the decrease of relative rotation speed though the clamp force is constant. This is because that the friction coefficient increases with the decreases of relative rotation speed of clutch drum and hub. In this simulation, at $0.29 \mathrm{~s}$, the relative rotation speed of clutch drum and hub reaches zero for a short moment as if they stick with each other and rotate together. Then, they slips with each other for a while and at $0.31 \mathrm{~s}$ they reach the same speed again and then slip with each other, at around $0.32 \mathrm{~s}$, they moves together again and the clutch becomes locked firmly. This intermittent lock and slip phenomenon is called stick-slip phenomenon[28], a phenomenon involving the intermittent 
slip and stick of clutch contact faces, which is affected by the dynamic characteristics of system and in return, aggravates the oscillation of the system. During the engagement process, there exist two kinds of vibration: the self-excited vibration and stick-slip phenomenon, and transient torque resulted, which affects the dynamic characteristics of driveline.
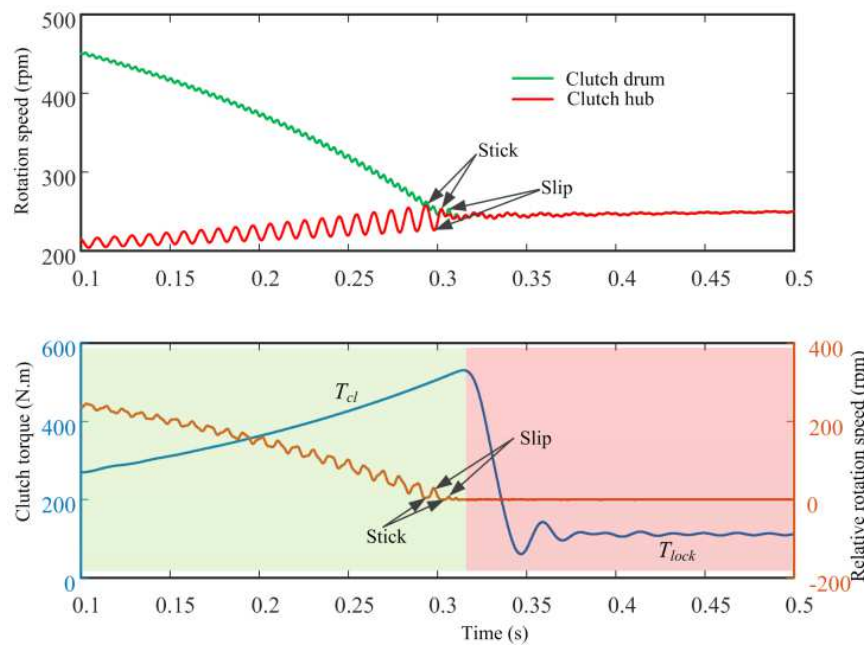

Figure 5 Dynamic characteristics of engaging clutch

\subsection{Gear set and torque coupling system modeling}

In the driveline, gear sets are often used as the torque amplifier as well as speed reducer, the dynamic characteristics of gear set also affects the dynamic characteristics of driveline, since the stick-slip phenomenon of the clutch is related to the friction characteristic of the clutch and the system mechanical dynamic characteristic[29, 30]. The fluctuation and impulsion in the rotation speed and torque have impact on the clutch stick-slip, that will aggravate the oscillation in the driveline and accelerates the degradation of frictional performance of clutch [27]. In the driveline, fluctuations can be caused by stiffness of shaft, time-varying mesh stiffness of gear set [29]. Therefore, in this study, when establishing the driveline model the stiffness and damping of the shafts, and time-varying mesh stiffness of gear set were considered.

The gear set transmits the torque when its teeth mesh with each other. When the gear set rotates, the meshing teeth pass through two states: double teeth contact area and single tooth contact area (Figure 6a). This periodic switching between these two states causes the mesh stiffness of gear set vary with the gear angular displacement, i.e., $\theta_{i}=\omega i t$, which is often referred to as time-varying mesh stiffness, $k_{m i k}(\mathrm{t})$. The mesh stiffness is $k_{s g}$ when the meshing gear is in the single tooth contact area, and is $k_{d b}$ when the meshing gears are in the double teeth contact area (Figure 6b).

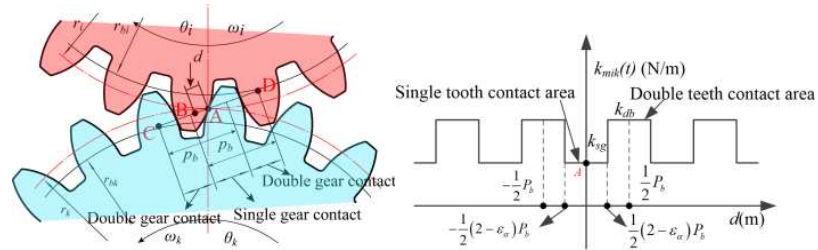

Figure 6 Time-varying mesh stiffness of gear set

The dynamic model of gear set is illustrated in Figure 7. In this model, the shafts and bearings are modeled as dampers to account for the compliance of these components. According to Newton's law, and the mathematical model of the gear set can be expressed with (15)-(20).

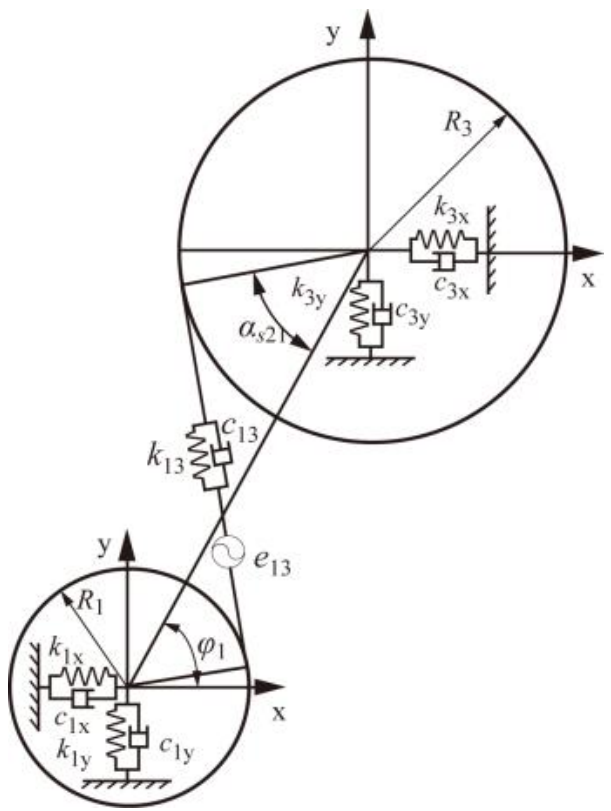

Figure 6 Model of a pair of meshing gears

$$
\begin{aligned}
& m_{1} \ddot{x}_{1}+c_{1 x} \dot{x}_{1}+k_{1 x} x_{1}+F x=0 \\
& m_{1} \ddot{y}_{1}+c_{1 y} \dot{y}_{1}+k_{1 y} y_{1}+F y=0 \\
& J_{1} \ddot{\theta}_{1}+F s R_{1}=T_{1} \\
& m_{3} \ddot{x}_{3}+c_{3 x} \dot{x}_{3}+k_{3 x} x_{3}-F x=0 \\
& m_{3} \ddot{y_{3}}+c_{3 y} \dot{y}_{3}+k_{3 y} y_{3}-F y=0 \\
& J_{3} \ddot{\theta}_{3}-F s R_{3}=-T_{3}
\end{aligned}
$$


where $m_{1}$ is the mass of gear $1, J_{1}$ is the moment of inertia of gear 1 , and $F i(i=x, y)$ are the forces in two directions of the Cartesian coordinate system. $k_{\mathrm{j}} \quad(j=i x, i y)$ are the stiffness of the bearings, $c_{\mathrm{j}}(j=i x$, iy) are the damping coefficients of the bearings, and $F_{s}$ is the dynamic mesh force between two gears.

$$
\begin{aligned}
& F_{s}=k_{13} \operatorname{dis}(t)+c_{13} \operatorname{dis}(t) \\
& F_{x}=F_{s} \sin \varphi \\
& F_{y}=F_{s} \cos \varphi \\
& c_{j}(t)=2 \xi_{g} \sqrt{k_{13} m_{1} m_{3} /\left(m_{1}+m_{3}\right)}
\end{aligned}
$$

Where, $\operatorname{dis}(t)$ is defined as

$$
\operatorname{dis}(t)=y_{1} \cos \varphi+x_{1} \sin \varphi+R_{1} \theta_{1}
$$$$
-y_{3} \cos \varphi-x_{3} \sin \varphi-R_{3} \theta_{3}-e_{13}(t)
$$

$\varphi=\varphi_{1}-\alpha_{s 21}$

$\xi_{g}$ is the damping ratio of gear set, $e_{13}(t)$ is the periodic profile error and $k_{13}(t)$ is the time-varying mesh stiffness and they can be expressed with Fourier series[31]. In our study, the 7-order Fourier series was used to approximate the time-varying mesh stiffness of the gear set.

$k_{13}(t)=\bar{k}_{13}^{--}\left\{1+\sum_{i=1}^{7} \hat{k}_{13 i} \sin \left(i \omega t+\hat{\phi_{13 i}}\right)\right\}$

$e_{13}(t)=e_{13 i}^{--}\left\{1+\sum_{j=1}^{7} \hat{e_{13 i}} \sin \left(i \omega t+\hat{\phi_{13 i}}\right)\right\}$

In DCT gearbox, two clutches transfer torque simultaneously while shifting and two pinions at the end of the two transfer shaft meshes with the differential gear ring simultaneously, which can be illustrated in figure 3. In previous literature, the torque coupling system were considered as lineal, however, it is nonlinear in practice, and the torque coupling system has important influence on the dynamic characteristics of the whole driveline[31]. Therefore, it is essential to build a dynamic model of the torque coupling system to investigate its dynamic characteristics. The torque coupling system consists of basic meshing gears. Therefore, based on the model of basic meshing gear set, the mathematical model of torque coupling system can be built.

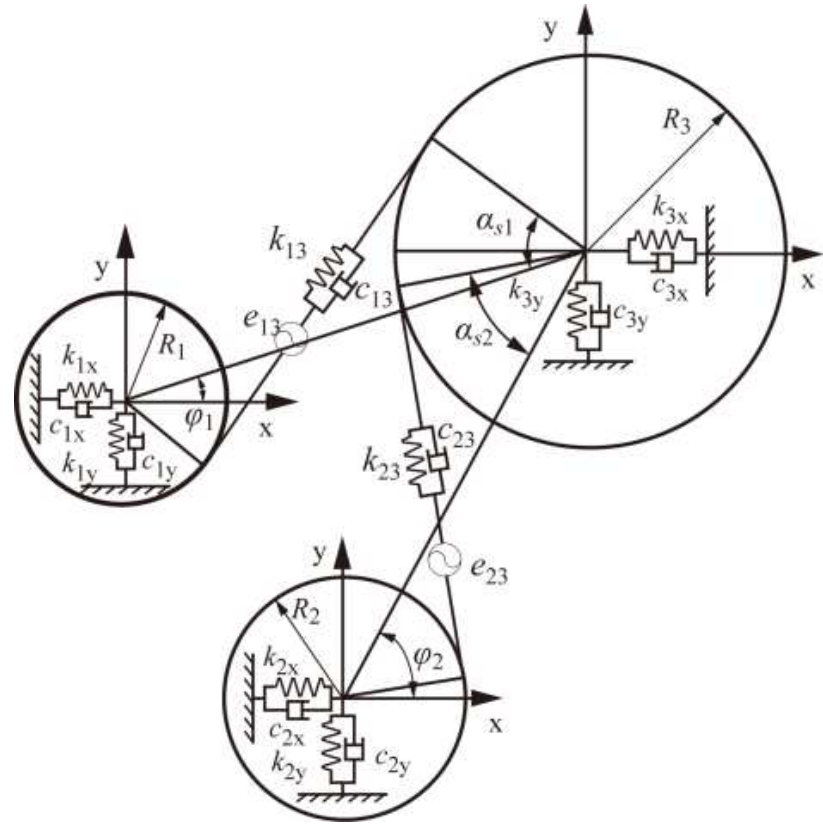

Figure 8 Dynamic model of gear system

Figure 8 presents the dynamic analysis of multi-mesh gear sets, the dynamic model of multi-mesh gear set can be expressed with (29)-(37).

$$
\begin{aligned}
& m_{1} \ddot{x}_{1}+c_{1 x} \dot{x}_{1}+k_{1 x} x_{1}=-F_{x 1} \\
& m_{1} \ddot{y}_{1}+c_{1 y} \dot{y}_{1}+k_{1 y} y_{1}=-F_{y 1} \\
& J_{1} \ddot{\theta}_{p 1}=T_{p 1}-F_{s 1} R_{1} \\
& m_{2} \ddot{x}_{2}+c_{2 x} \dot{x}_{2}+k_{2 x} x_{2}=-F_{x 2} \\
& m_{2} \ddot{y_{2}}+c_{2 y} \dot{y}_{2}+k_{2 y} y_{2}=-F_{y 2} \\
& J_{2} \ddot{\theta}_{p 2}=T_{p 2}-F_{s 2} R_{2} \\
& m_{3} \ddot{x}_{3}+c_{3 x} \dot{x}_{3}+k_{3 x} x_{3}=F_{x 1}+F_{x 2} \\
& m_{3} \ddot{y}_{3}+c_{3 y} \dot{y}_{3}+k_{3 y} y_{3}=F_{y 1}+F_{y 2} \\
& J_{3} \ddot{\theta}_{3}=F_{s 1} R_{1}+F_{s 2} R_{2}-T_{p 3}
\end{aligned}
$$

Where, $F_{i}(i=x 1, x 2, x 3, y 1, y 2, y 3)$ are the forces in the three directions of the Cartesian coordinate system. $F_{s i}(i=$ $1,2)$ are the dynamic mesh forces between the gear sets. $J_{i}$ $(i=1,2,3)$ are the moments of inertia of the three gears. $T_{i}$ $(i=p 1, p 2, p 3)$ are the torques that were loaded on the three gears.

\subsection{Resistive force acting on the entire vehicle}

When the vehicle runs, resistive forces act on it, some come into play from the start of the motion whereas others build up with speed. The power generated by the engine is 
mainly consumed by the rolling resistance force, air resistance, gradient, and acceleration of rotating components in the driveline[24]. The total resistive torque acting on the running vehicle can be calculated by equation (38).

$T_{L}=\left(F_{f}+F_{w}+F_{i}+F_{j}\right) r_{W}$
Where, $m$ is the mass of the vehicle. $g$ is the gravitational acceleration. $f$ is the rolling resistance coefficient, $\theta$ is the slope angle, $C_{D}$ is the aerodynamic force coefficient, $A$ is the project area of the vehicle, $\rho$ is the density of the air, $v$ is the velocity, $\delta$ is the acceleration coefficient, and $r_{W}$ is the radius of wheel.

$F_{f}=m g f \cos \theta, \quad F_{w}=0.5 C_{D} A \rho v^{2}, \quad F_{i}=m g \sin \theta, \quad F_{j}=\delta m d v / d t$

\subsection{Vehicle driveline modeling}

To obtain the dynamic characteristics of the DCT during shift process, the entire vehicle propulsion system needs to be modeled. A schematic of the vehicle driveline system is shown in Figure 9.

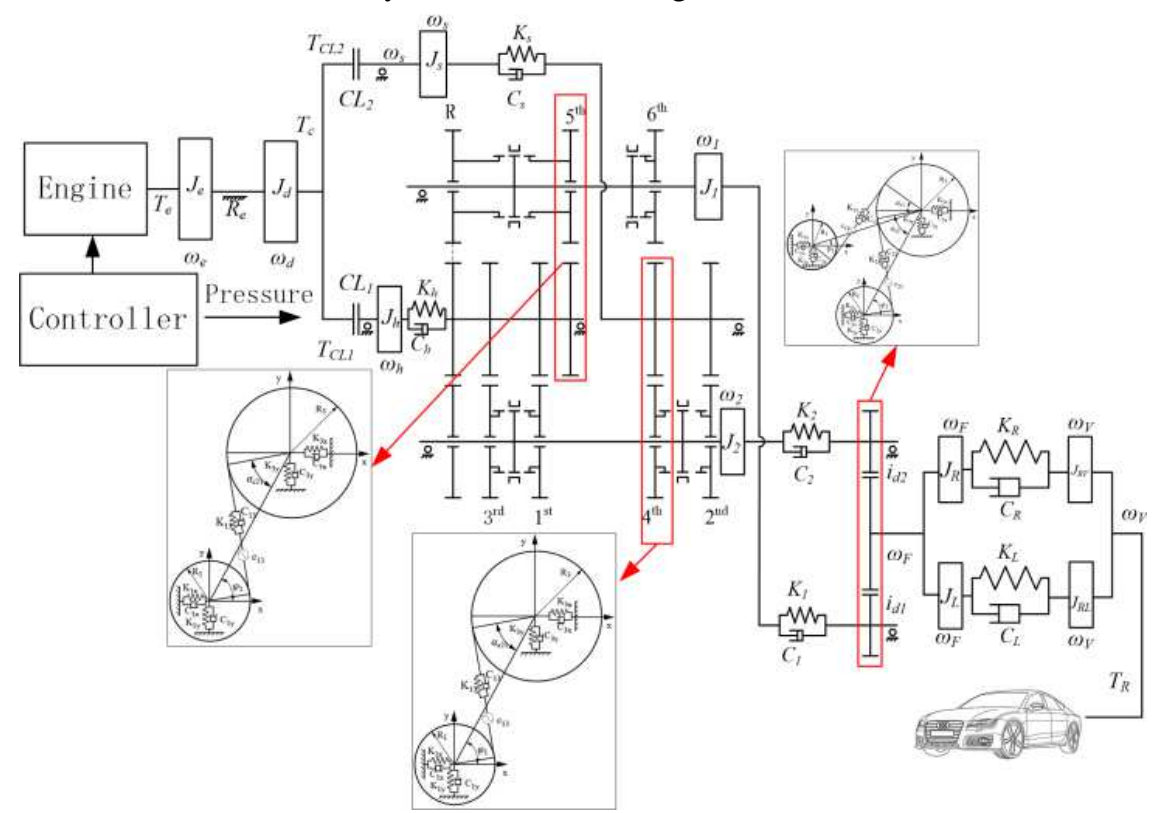

Figure 9 Schematic of the vehicle powertrain system

In this model, two assumptions were made:

The influence of the temperature on the transmission efficiency was ignored.

The time delay of the mechanism and hydraulic actuation system was ignored.

Based on these assumptions, the schematic can be simplified, as shown in Figure 10.

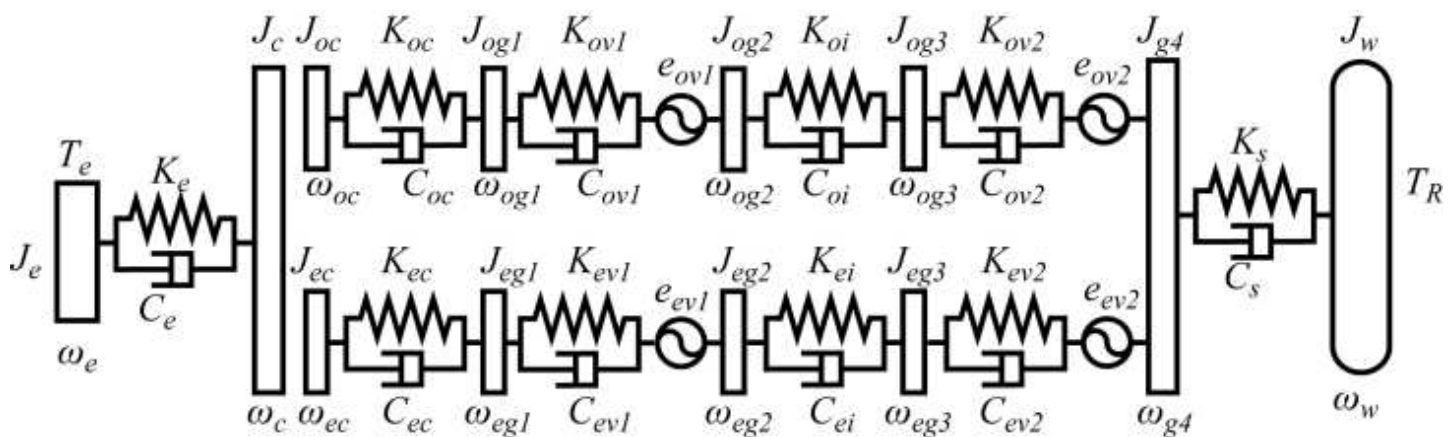

Figure 10 Dynamic model of vehicle powertrain system

Based on the developed engine model, clutch model, and gearbox model, the entire driveline model can be established 
with equations (39-49).

$J_{e} \ddot{\theta}_{e}=-C_{e} \dot{\theta}_{e}+C_{e} \dot{\theta}_{d}-K_{e} \theta_{e}+K_{e} \theta_{d}+T_{e}$

$J_{c} \ddot{\theta}_{c}=C_{e} \dot{\theta}_{e}-\left(C_{e}+k_{o d} \mu_{o s} F_{o d} N_{o d} R_{o d}+k_{e v} \mu_{e s} F_{e v} N_{e v} R_{e v}\right) \dot{\theta}_{c}+k_{o d} \mu_{o s} F_{o d} N_{o d} R_{o d} \dot{\theta}_{o c}$

$+k_{e v} \mu_{e s} F_{e v} N_{e v} R_{e v} \theta_{e c}+K_{e} \theta_{e}-K_{e} \theta_{c}-\mu_{o s} F_{o d} N_{o d} R_{o d}-\mu_{e s} F_{e v} N_{e v} R_{e v}$

$J_{o c} \ddot{\theta}_{o c}=k_{o d} F_{o d} N_{o d} R_{o d} \dot{\theta}_{c}-\left(k_{o d} F_{o d} N_{o d} R_{o d}+C_{o c}\right) \dot{\theta_{o c}}+C_{o c} \theta_{o g 1}-K_{o c} \theta_{o c}$

$+K_{o c} \theta_{o g 1}+\mu_{o s} F_{o d} N_{o d} R_{o d}$

$J_{o g 1} \theta_{o g 1}=C_{o c} \dot{\theta_{o c}}-\left(C_{o c}+C_{o v 1} R_{o g 1}\right) \dot{\theta}_{o g 1}+C_{o v 1} R_{o g 2} \theta_{o g 2}+K_{o c} \theta_{o c}-\left(K_{o c}+K_{o v 1} R_{o g 1}\right) \theta_{o g 1}+K_{o v 1} R_{o g 2} \theta_{o g 2}$

$-K_{o v 1} y_{o g 1} \cos \varphi_{o v 1}-K_{o v 1} x_{o g 1} \sin \varphi_{o v 1}+K_{o v 1} y_{o g 2} \cos \varphi_{o g 2}+K_{o v 1} x_{o g 2} \sin \varphi_{o v 1}-C_{o v 1} y_{o g 1} \cos \varphi_{o v 1}$

$-C_{o v 1} x_{o g 1} \sin \varphi_{o v 1}+C_{o v 1} y_{o g 2} \cos \varphi_{o v 1}+C_{o v 1} x_{o g 2} \sin \varphi_{o v 1}+K_{o v 1} e_{o v 1}+C_{o v 1} e_{o v 1}$

$J_{o g 2} \theta_{o g 2}^{\ddot{*}}=C_{o v 1} R_{o g 1} \theta_{o g 1}-\left(C_{o v 1} R_{o g 2}+C_{o i}\right) \theta_{o g 2}^{\cdot}+C_{o i} \theta_{o g 3}+K_{o v 1} R_{o g 1} \theta_{o g 1}-\left(K_{o v 1} R_{o g 2}+K_{o i}\right) \theta_{o g 2}$

$+K_{o i} \theta_{o g 3}+C_{o v 1} y_{o g 1} \cos \varphi_{o v 1}+C_{o v 1} x_{o g 1} \sin \varphi_{o v 1}-C_{o v 1} y_{o g 2} \cos \varphi_{o v 1}+C_{o v 1} x_{o g 2} \sin \varphi_{o v 1}-C_{o v 1} e_{o v 1}$

$+K_{o v 1} y_{o g 1} \cos \varphi_{o v 1}+K_{o v 1} x_{o g 1} \sin \varphi_{o v 1}-K_{o v 1} y_{o g 2} \cos \varphi_{o v 1}-K_{o v 1} x_{o g 2} \sin \varphi_{o v 1}-K_{o v 1} e_{o v 1}$

$J_{o g 3} \theta_{o g 3}^{\prime \prime}=C_{o i} \theta_{o g 2}-\left(C_{o i}+C_{o v 2} R_{o g}\right) \theta_{o g 3}+C_{o v 2} R_{g 4} \theta_{g 4}+K_{o i} \theta_{o g 2}-\left(K_{o i}+K_{o v 2} R_{o g 3}\right) \theta_{o g 3}$

$+K_{o v 2} R_{g 4} \theta_{g 4}-C_{o v 2} y_{o g 3} \cos \varphi_{o v 2}-C_{o v 2} x_{o g 3} \sin \varphi_{o v 2}+C_{o v 2} y_{g 4} \cos \varphi_{o v 2}+C_{o v 2} x_{g 4} \sin \varphi_{o v 2}$

$+C_{o v 2} e_{o v 2}-K_{o v 2} y_{o g 3} \cos \varphi_{o v 2}-K_{o v 2} x_{o g 3} \sin \varphi_{o v 2}+K_{o v 2} y_{g 4} \cos \varphi_{o v 2}+K_{o v 2} x_{g 4} \sin \varphi_{o v 2}+K_{o v 2} e_{o v 2}$

$J_{e c} \ddot{\theta_{e c}}=k_{e v} F_{e v} N_{e v} R_{e v} \dot{\theta}_{c}-\left(k_{e v} F_{e v} N_{e v} R_{e v}+C_{e c}\right) \dot{\theta}_{e c}+C_{e c} \dot{\theta}_{e g 1}-K_{e c} \theta_{e c}+K_{e c} \theta_{e g 1}+\mu_{e s} F_{e v} N_{e v} R_{e v}$

$J_{e g 1} \theta_{e g 1}^{\ddot{\prime}}=C_{e c} \dot{\theta_{e c}}-\left(C_{e c}+C_{e v 1} R_{e g 1}\right) \theta_{e g 1}^{\dot{1}}+C_{e v 1} R_{e g 2} \dot{\theta}_{e g 2}+K_{e c} \theta_{e c}-\left(K_{e c}+K_{e v 1} R_{e g 1}\right) \theta_{e g 1}+K_{e v 1} R_{e g 2} \theta_{e g 2}$

$-K_{e v 1} y_{e g 1} \cos \varphi_{e v 1}-K_{e v 1} x_{e g 1} \sin \varphi_{e v 1}+K_{e v 1} y_{e g 2} \cos \varphi_{e g 2}+K_{e v 1} x_{e g 2} \sin \varphi_{e v 1}-C_{e v 1} y_{e g 1} \cos \varphi_{e v 1}$

$-C_{e v 1} x_{e g 1} \sin \varphi_{e v 1}+C_{e v 1} y_{e g 2} \cos \varphi_{e v 1}+C_{e v 1} x_{e g 2} \sin \varphi_{e v 1}+K_{e v 1} e_{e v 1}+C_{e v 1} e_{e v 1}$

$J_{e g 2} \theta_{e g 2}^{\ddot{\prime}}=C_{e v 1} R_{e g 1} \theta_{e g 1}-\left(C_{e v 1} R_{e g 2}+C_{e i}\right) \theta_{e g 2}^{\dot{2}}+C_{e i} \dot{\theta}_{e g 3}^{\dot{3}}+K_{e v 1} R_{e g 1} \theta_{e g 1}-\left(K_{e v 1} R_{e g 2}+K_{e i}\right) \theta_{e g 2}$

$+K_{e i} \theta_{e g 3}+C_{e v 1} y_{e g 1} \cos \varphi_{e v 1}+C_{e v 1} x_{e g 1} \sin \varphi_{e v 1}-C_{e v 1} y_{e g 2} \cos \varphi_{e v 1}+C_{e v 1} x_{e g 2} \sin \varphi_{e v 1}-C_{e v 1} e_{e v 1}$

$+K_{e v 1} y_{e g 1} \cos \varphi_{e v 1}+K_{e v 1} x_{e g 1} \sin \varphi_{e v 1}-K_{e v 1} y_{e g 2} \cos \varphi_{e v 1}-K_{e v 1} x_{e g 2} \sin \varphi_{e v 1}-K_{e v 1} e_{e v 1}$

$J_{e g 3} \theta_{e g 3}^{\ddot{\prime}}=C_{e i} \theta_{e g 2}-\left(C_{e i}+C_{e v 2} R_{e g 3}\right) \dot{\theta}_{e g 3}+C_{e v 2} R_{g 4} \dot{\theta}_{g 4}+K_{e i} \theta_{e g 2}-\left(K_{e i}+K_{e v 2} R_{e g 3}\right) \theta_{e g 3}$

$+K_{e v 2} R_{g 4} \theta_{g 4}-C_{e v 2} y_{e g 3} \cos \varphi_{e v 2}-C_{e v 2} x_{e g 3} \sin \varphi_{e v 2}+C_{e v 2} y_{g 4} \cos \varphi_{e v 2}+C_{e v 2} x_{g 4} \sin \varphi_{e v 2}$

$+C_{e v 2} e_{e v 2}-K_{e v 2} y_{e g 3} \cos \varphi_{e v 2}-K_{e v 2} x_{e g 3} \sin \varphi_{e v 2}+K_{e v 2} y_{g 4} \cos \varphi_{e v 2}+K_{e v 2} x_{g 4} \sin \varphi_{e v 2}+K_{e v 2} e_{e v 2}$ 


$$
\begin{aligned}
& J_{g 4} \ddot{\theta}_{g 4}=C_{o v 2} R_{o g 3} \dot{\theta_{o g} 3}+C_{e v 2} R_{e g 3} \dot{\theta}_{e g 3}-\left(C_{o v 2} R_{g 4}+C_{e v 2} R_{g 4}+C_{s}\right) \dot{\theta_{g} 4}+C_{s} \dot{\theta}_{w}+K_{o v 2} R_{o g 3} \theta_{o g 3} \\
& +K_{e v 2} R_{e g 3} \theta_{e g 3}-\left(K_{o v 2} R_{g 4}+K_{e v 2} R_{g 4}+K_{s}\right) \theta_{g 4}+K_{s} \theta_{w}+C_{o v 2} y_{o g 3} \cos \varphi_{o v 2}+C_{o v 2} x_{o g 3} \sin \varphi_{o v 2} \\
& -C_{o v 2} e_{o v 2}+C_{e v 2} y_{e g 3} \cos \varphi_{e v 2}-C_{e v 2} x_{e g 3} \sin \varphi_{e v 2}-C_{e v 2} e_{e v 2}-\left(C_{o v 2} \cos \varphi_{o v 2}+C_{e v 2} \cos \varphi_{e v 2}\right) y_{g 4} \\
& -\left(C_{o v 2} \sin \varphi_{o v 2}+C_{e v 2} \sin \varphi_{e v 2}\right) x_{g 4}+K_{o v 2} \mathrm{y}_{o g 3} \cos \varphi_{o v 2}+K_{o v 2} x_{o g 3} \sin \varphi_{o v 2}-K_{o v 2} e_{o v 2} \\
& +K_{e v 2} y_{e g 3} \cos \varphi_{e v 2}-K_{e v 2} x_{e g 3} \sin \varphi_{e v 2}-K_{e v 2} e_{e v 2}-\left(K_{o v 2} \cos \varphi_{o v 2}+K_{e v 2} \cos \varphi_{e v 2}\right) y_{g 4} \\
& -\left(K_{o v 2} \sin \varphi_{o v 2}+K_{e v 2} \sin \varphi_{e v 2}\right) x_{g 4} \\
& J_{w} \dot{\theta}_{w}=C_{s} \dot{\theta}_{g 4}-C_{s} \dot{\theta}_{w}+K_{s} \theta_{g 4}-K_{s} \theta_{w}-T_{R}
\end{aligned}
$$

Where, $\theta_{i}(i=e, c, o c, o g 1, o g 2, o g 3, e c, e g 1, e g 2, e g 3, g 4, w)$ are the rotated angles of the components, $J_{i}(i=e, c, o c, o g 1$, $o g 2, o g 3, e c, e g 1, e g 2, e g 3, g 4, w)$ are the inertia of the components, $K_{i}(i=e, o c, o v 1, o i, o v 2, e c, e v 1, e i, e v 2, s)$ are the stiffness of shafts, $C_{i}(i=e, o c, o v 1, o i, o v 2, e c, e v 1, e i, e v 2, s)$ are the equivalent damping of shafts, $T_{e}$ is the engine torque, $T_{R}$ is the resistance torque. $x_{i}(i=o g 1, o g 2, o g 3, e g 1, e g 2, e g 3, g 4)$ are the displacements of gears in $x$ axis direction, $y_{i}(i=$ $o g 1, o g 2, o g 3, e g 1, e g 2, e g 3, g 4)$ are the displacement of gears in y axis direction, $\varphi_{i}(i=o v 1, o v 2, e v 1, e v 2)$ is the installation angle of gears.

\subsection{Shift control logic}

The time of shift depends on the states of driveline. Generally, in automatic transmission, the shifts depend on the velocity of vehicle and the opening position of the throttle [22]. Thus, a shifting controller, which receives the states of the driveline components and makes the decision to change the gear is needed. In this study, the driveline route was built in the MATLAB/Simulink environment based on differential equations. The shifting controller was built with a MATLAB/Stateflow chart. The model receives the throttle pedal signal and velocity of the vehicle and outputs the clutch pressure. The shift control flow can be seen in figure 11.

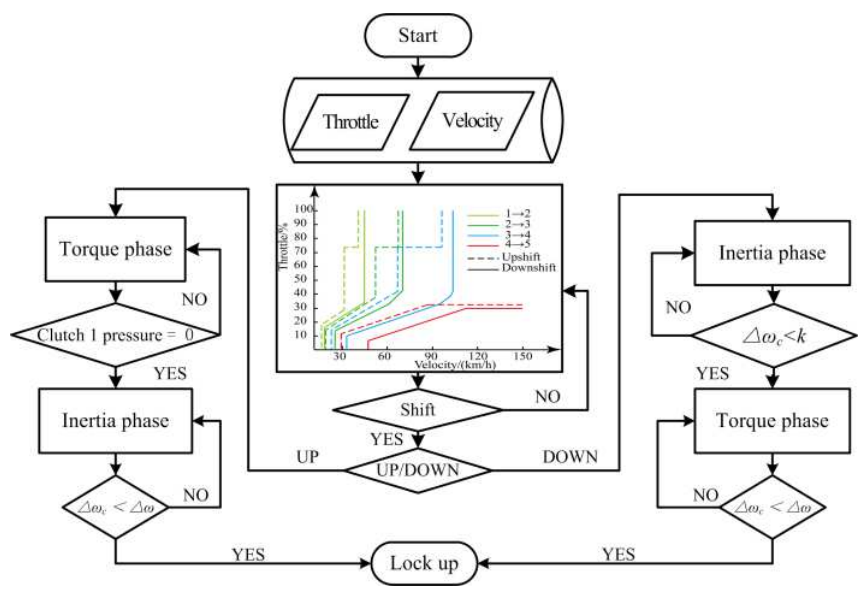

Figure 11 Gearshift logic

\subsection{Clutch control}

As mentioned before, the change rate of two input torque is vital to the quality of shift, which means that the two clutches should be controlled coordinately. The profile of the pressure of clutch can be illustrated with figure 12 . During the shifting process, the adjusted torque of the engine is controlled by a PID controller, which simulates the spark and injection advance and the force act on the clutch also controlled by PID controllers.

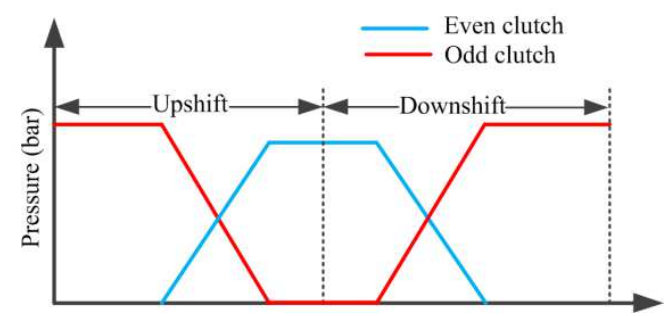

Figure 12 Clutch pressure control profile during shifting

\subsection{Simulation results}

In this study, upshift from $1^{\text {st }}$ to $2^{\text {nd }}$ gear processes was simulated with a sedan whose main parameters are listed in the Appendix. In this model, the time-varying mesh stiffness was considered and the finite state machine was used to calculate the shift time, thus the solver sample time of the solver was set at the order of $10^{-7}$, which is shorter than the sample time of the speed sensor in practice. Therefore, to validate the mathematical model of driveline 
and compare with the test data, which was obtained from the sensors installed on the vehicle, a filter was designed based on the sample time of the sensors on the vehicle. Figure 13 presents the simulation result with the filter.

(a)

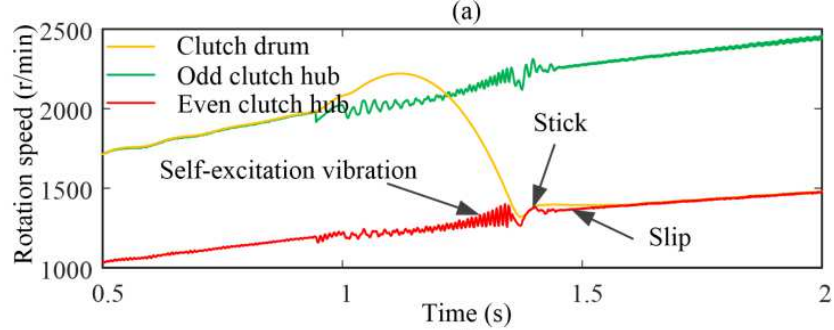

(b)

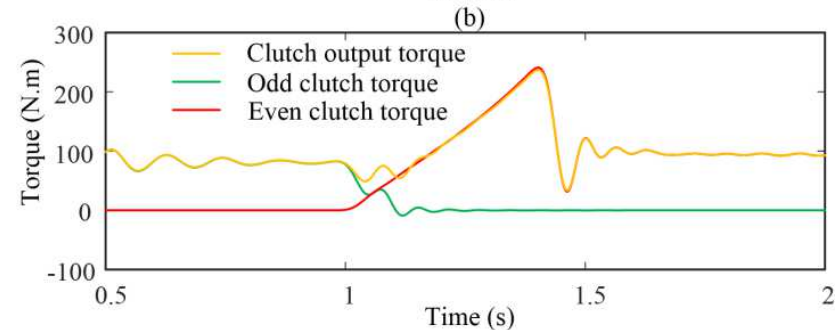

Figure 13 Simulation result of shift

Upper graphic in figure 13 presents the ration speed of clutch drum, odd and even clutch hub. Lower graphic in figure 13 presents the output torque of clutch, the torque transmitted by odd and even clutch. Before shift, the clutch drum rotates together with odd clutch hub, at $0.93 \mathrm{~s}$, the vehicle started to shift, odd clutch started slipping, rotation speed of clutch drum increased at first, and then decreased because of the increase of torque transmitted by even clutch. At $1.37 \mathrm{~s}$, the shift process finished. During this process, the rotation speed of odd and even clutch hub suffered oscillations. At the beginning of shift, odd clutch began slipping, the clutch switched from locked to slipping state, and the torque transmitted by the clutch also changed. The torque transmitted by odd clutch decreased, while the torque transmitted by the even clutch did not increase as fast because of the friction coefficient is small when the relative rotation speed between the clutch drum and even clutch hub is large, therefore, the clutch drum accelerates for a while. Due to the sudden change of the torque transmitted by odd clutch at the beginning of shift, the ration speed of odd clutch hub suffers oscillation. At the end of shift, the oscillation amplitude of rotation speed of even clutch hub became higher and higher as the even clutch engages, this phenomenon named self-excited vibration was caused by negative gradient variation of the friction coefficient, as mentioned in section 2.2. When the even clutch engages, the torque transmitted by even clutch increased rapidly as the friction coefficient increases with the decrease of relative rotation speed and the increase of clutch pressure. When the clutch became locked, the torque transmitted by the clutch switched from $T_{c l}$ to $T_{\text {lock }}$, which also aggravates the oscillation of rotation speed of even clutch. And at the end of shift, stick-slip phenomenon also appeared due to the torque oscillation.

\subsection{Experiment and model validation}

To validate the simulation result, an experiment was done with a car equipped with DCT and a data recorder device as shown in figure $14 \mathrm{a}, \mathrm{b}$ The data recorder device is used to collect the data of the vehicle. The vehicle was tested for a long distance at different condition.

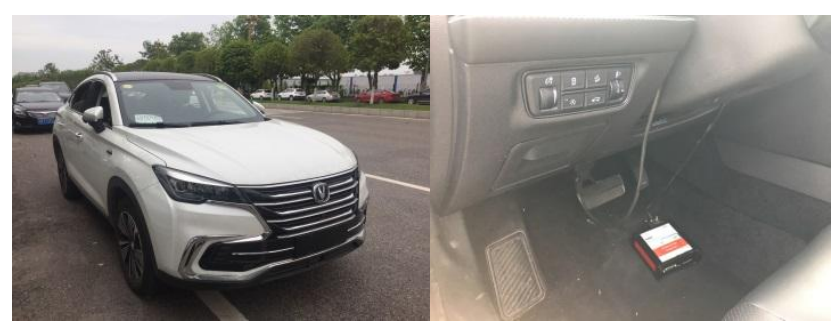

(a)

(b)

Figure 14 Tested vehicle and data recorder

Figure 15 shows the comparison result between the simulation data and test data. And, the time at the horizontal axis is calculated with the sample time of the recorder device and the number of the sample points.

The dash lines show the tested data of a shift from $1^{\text {st }}$ to $2^{\text {ed }}$, which was obtained from the data recorder device. It can be seen that the shift begins at $0.2 \mathrm{~s}$ and finishes at $0.54 \mathrm{~s}$. At beginning of shift, when the odd clutch begins to slip, the rotation speed of odd clutch hub suffers oscillations due to the transient change of the torque, so does the even clutch hub due to the torque coupling device. The rotation speed of the clutch drum accelerates for a while, then it starts to decrease to synchronize with the even clutch hub. At the end of the shift, the rotation speed of even clutch hub suffers oscillations at $0.47 \mathrm{~s}$ and the amplitude of the oscillation becomes higher and higher as the clutch engages. When the shift finishes, the clutch runs in the micro-slip state. The comparison result between test and simulation data shows that there is a little difference in shift time between the simulation and test data, which is caused by the actuator and other factors, because in the simulation, the actuator is built with ideal assumption. The simulation data has the same feature with the test data, especially at the beginning and end of the shift, both presents the oscillations in the rotation speed of clutch hub, and the change trend of the simulation data is consistent 
with the test data, which can be thought that the model is credible.

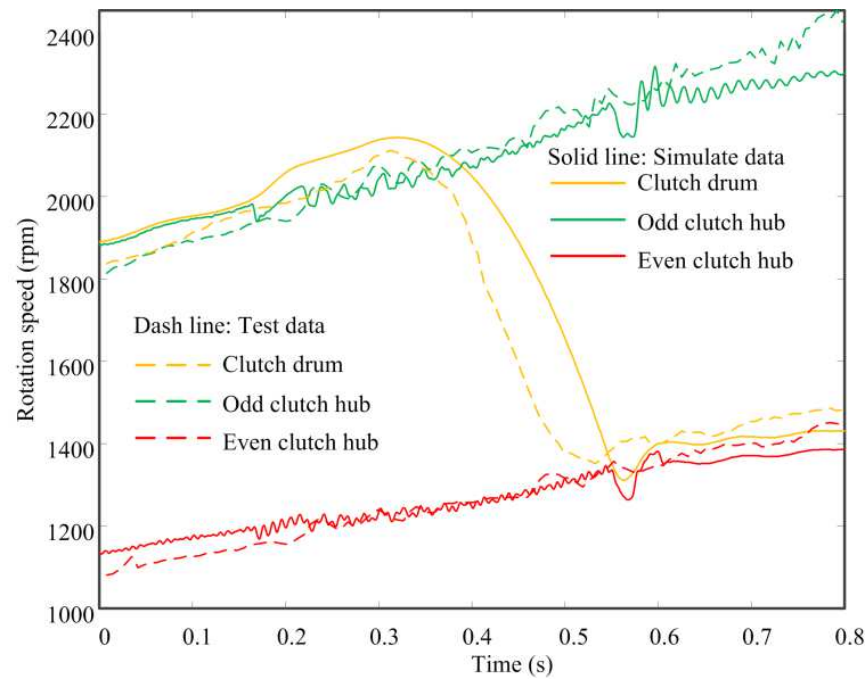

Figure 15 Comparison between the simulation and test

\section{Variation of dynamic characteristics of driveline along with the service life}

Throughout the service life cycle, components in the driveline will suffer degradation process, so does the wet clutch. The frictional characteristic degrades with the engagement cycles. A notable feature is that the static friction coefficient and negative gradient of friction coefficient change with clutch engagement cycles [27], as shown in figure 16.

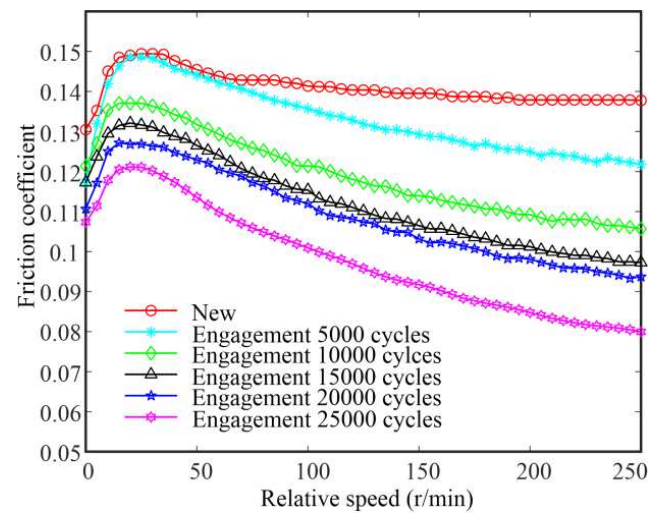

Figure 16 Friction coefficient of wet clutch at different stages

As the service time increases, the torque transmission performance of clutch decreases[25-27], Thus the dynamic characteristics of driveline during shift process varies with the service life of clutch, figure 17 shows the dynamic characteristics of driveline with different frictional characteristics.
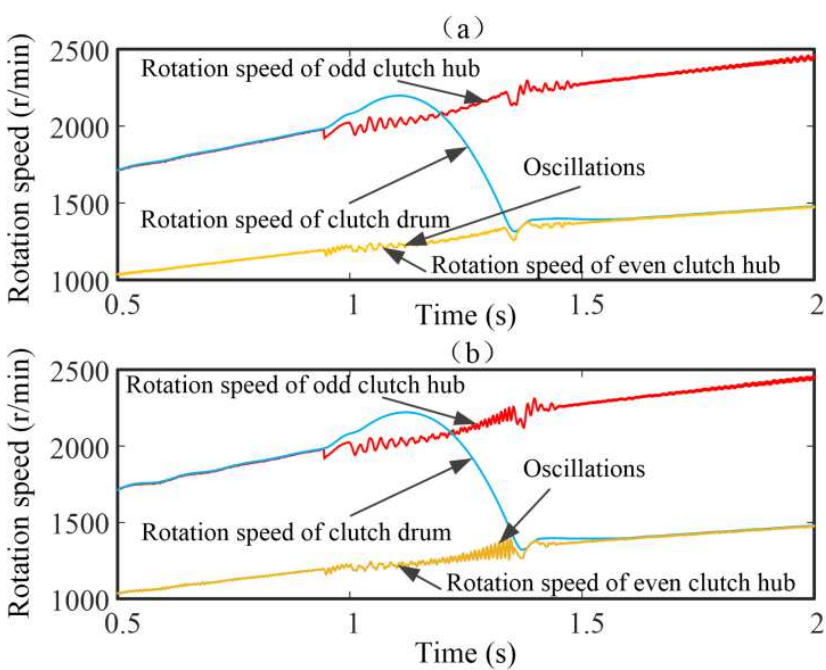

Figure 17 Effect of negative gradient of friction coefficient on dynamic characteristics of driveline

Figure 16 shows the dynamic characteristics of shift process with different negative gradient of friction coefficient. Upper graph shows the result with negative gradient of friction coefficient, $k=-0.00001$, figure $16 \mathrm{~b}$ shows the shift process with $k=-0.000015$. From this figure, it can be seen that, both start time of shift are same, at $0.94 \mathrm{~s}$, while the end time of shift process is a little different. When $k=-0.00001$, the end time of shift process is $1.35 \mathrm{~s}$, while the end time of shift process is $1.37 \mathrm{~s}$ when $k=-0.000015$. This means that when the negative gradient is small, the shift time is long due to the degradation of clutch frictional performance. Another significant difference between these two cases is that the amplitude of oscillations in the driveline. The amplitude of oscillation in figure $16 \mathrm{~b}$ is higher than that in figure $16 \mathrm{a}$, which means that the driveline suffers severe oscillation when the clutch frictional performance decreases. Comparison in figure 16 shows that, friction characteristics of clutch affects dynamic characteristics of driveline during shift process, small negative gradient of friction coefficient will result in severe oscillation and long shift time.

\section{Effects of controllable parameters on the dynamic characteristics of driveline during shift process}

The dynamic characteristics of driveline changes with the variation of frictional characteristics, which varies with service life. As the service life increases, the frictional characteristics decreases, and the driveline will suffer severe oscillations. To attenuate this side effect by adjust 
controllable parameters, degradation law of friction characteristics should be obtained first.

To obtain the friction coefficient of wet clutch at different stage of service life, thousands of tests need to be performed, which is an time-consuming task. Although the SAE\#2 rig can be used to test the friction coefficient of wet clutch, it is an acceleration-life test, can not reflect practical working states of wet clutch installed on the vehicle. And to obtain the practical degradation law of wet clutch, a test rig (figure 18a) with dual clutch transmission was established to simulate the working states of vehicle. And the clutches, which were taken from the new, half-new and old vehicles were tested (figure 18b) with the rig. The torque transmitted by clutch and rotation speed of clutch drum and hub were collected, the variation of friction coefficient with relative rotation speed under practical working states were obtained with repetitive simulation of shift and start-up process on this rig. Service life of wet clutch could be deduced based on the consistency of service life of components in a vehicle. The mean service life of the vehicle was obtained from the vehicle manufactory, and according to the statistical result from the manufactory, a wet clutch could sustain the vehicle working 300000 kilometers before it is replaced or failure. With this data, the total engagement cycles of wet clutch can be deduced, and the degradation law of wet clutch can also be deduced with the test data. Figure 10c shows the UDDS cycle and the gear state of the vehicle, which we studied. In this study, the vehicle shift from 1st to 2ed, according to shift logic and the structure of the gearbox, the even clutch engages 58 times in one UDDS cycle, thus total number of wet clutch engagement cycles can be deduced. The stages of wet clutch can be divided according to the condition of the vehicle, in this study, the lifespan of wet clutch were divided into three stages: early stage, metaphase, and final stage, as shown in figure 18d.

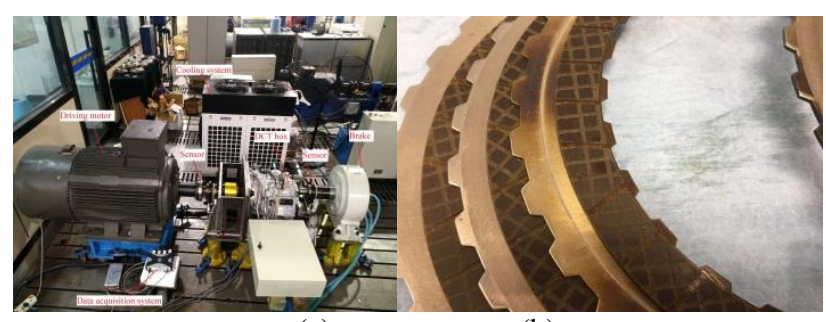

(a)

(b)

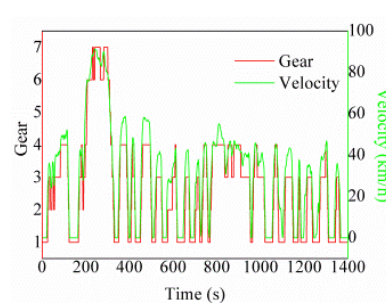

(c)

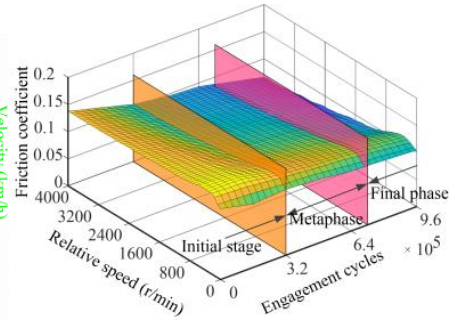

(d)
Figure 18 (a) Clutch friction plate; (b) test rig; (c) UDDS cycles and gear states of vehicle; (d) Division of the service life stages of wet clutch

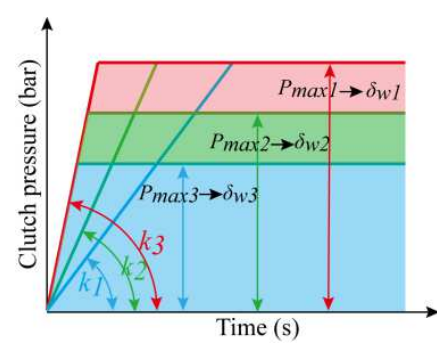

(a)

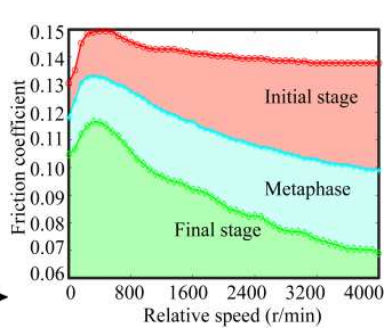

(b)
Figure 19 (a) Rising rate of hydraulic pressure and maximum pressure; (b) friction coefficient at different stages

As mentioned before, the driveline will suffer self-excitation and stick-slip during shift process. And the self-excitation can be attenuated by controlling the hydraulic pressure of wet clutch, and the stick-slip can be attenuated by making the clutch run in micro-slip state with proper threshold [32-35]. The threshold, which is determined by the maximum pressure of wet clutch at the end of shift process, as shown in figure 19a. And both the changing rate of the pressure and the maximum pressure can be controlled. Therefore, to obtain good performance of shift process, the effect of these two controllable parameters on the dynamic characteristics should be investigated. In this study, the rising rate of pressure was divided into three ranges based on the statistical data from the manufactory, so was the maximum of pressure at the end of shift process, as shown in figure 19a. And the lifespan of clutch was divided into three stages based on the engagement cycle as shown in figure $19 b$.

During shift process, the driveline suffers two kinds of oscillations: self-excitation and stick-slip during shift process. The magnitude of the oscillation can be defined as the degree of deviation from the equilibrium position. Therefore, when analyzing the clutch self-excitation oscillation, the standard deviation of the rotation speed of clutch hub can be chosen as an index. The standard deviation of rotation speed of clutch hub can be expressed 
by equation (51). And the number of stick-slip can be chosen as an index to evaluate the stick-slip phenomenon. Since the micro-slip method is adopted, when the clutch runs at micro-slip state, extra frictional loss will be caused, Therefore, frictional loss should be calculated to ensure that the heat caused by the frictional loss does limited side effect on the clutch. Also, when shift, the shift time should be also taken into consideration. Therefore, in this study, the standard deviation of rotation speed of clutch hub, the number of stick-slip, frictional loss and synchronizing time were chosen as the indexes.

$$
\begin{aligned}
& s t d=\left((\omega(t)-\overline{\omega(t)})^{2} / T\right)^{1 / 2} \\
& W_{f}=\int_{t_{0}}^{t_{1}} T_{c l i}\left(\Delta \omega_{c i}\right) d t
\end{aligned}
$$

Where, $T$ is the number of sample times, $t$ is the sample time, $\overline{\omega(t)}$ is the mean value of rotation speed of clutch hub at the moment of sampling. This value can be obtained by the least squares method, $W_{f}$ is the frictional loss, $t_{o}$ is the start time of shift and $t_{l}$ is the end time of shift, $T_{c l i}$ is the torque transmitted by clutch, $\Delta \omega_{c i}$ is the relative rotation speed between clutch drum and hub during shift process.

\section{Early stage}

At Early stage, the static friction coefficient and the gradient of friction coefficient is high, as shown in figure $19 \mathrm{~b}$, which means that the frictional characteristic of clutch is in a good condition, and figure 20 shows the statistical result of shift process.

Figure 20a shows the statistical result of shift time. Figure 20b shows the standard deviation of rotation speed of clutch hub. Figure 20c shows the result of frictional loss during shift process, and the pink plane represents the maximum permitted value of frictional loss during shift process. Figure $20 \mathrm{~d}$ shows the statistical result of number of stick-slip phenomenon. It can be seen from figure 20a that, the shift time decreases as the rising rate of clutch pressure increases, and the threshold of micro-slip (maximum pressure) at the end of shift has little effect on the shift time. Figure $20 \mathrm{~b}$ shows that, at early stage of clutch service life, the oscillations in the driveline vary with the rising rate of clutch pressure and threshold of micro-slip. When the clutch pressure increases fast, the driveline suffers intense oscillation. When the pressure rises at slow speed, the oscillation in the driveline becomes intense with the decrease of threshold of micro-slip at first, and then, dose not change with the variation of threshold of micro-slip. When the clutch pressure rises at fast speed, the oscillation in the driveline becomes intense as the threshold of micro-slip decreases at first, and then decreases as the threshold of micro-slip increases. Figure $20 \mathrm{c}$ shows that friction loss increases with the increase of threshold of micro-slip, shift with slow rising speed of pressure and large threshold of micro-slip results in more frictional loss. Figure 20d shows that, the propensity of stick-slip phenomenon decreases as the threshold of micro-slip increases. From above all, it can be concluded that, at early stage of clutch service life, slow rising speed of pressure and medium threshold of micro-slip should be adopted to obtain good performance of shift.
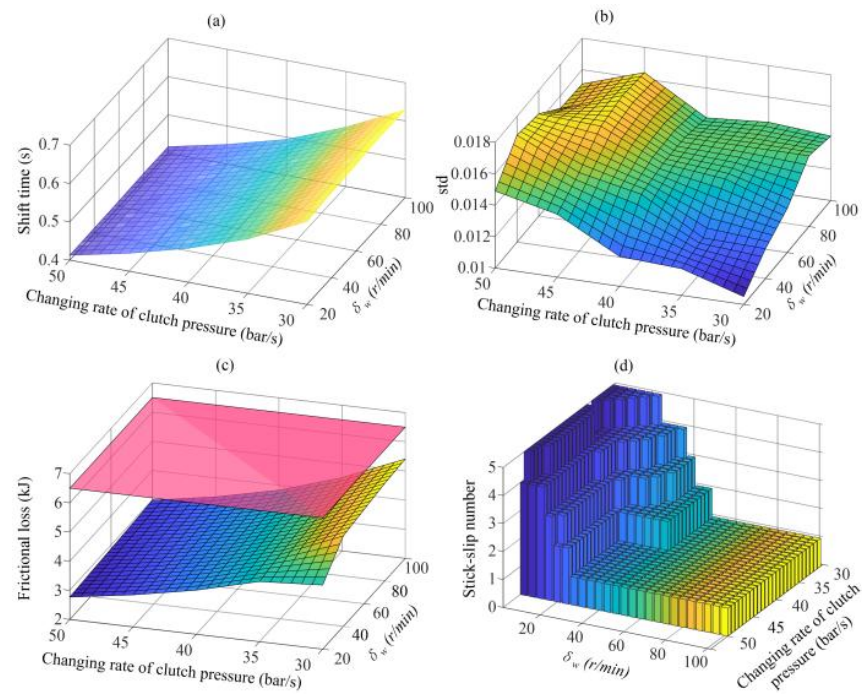

Figure 20 Coupling effect of controllable parameters on dynamic characteristics of driveline at early stage

\section{Metaphase}

Compared with the early stage, the static friction coefficient and gradient of friction coefficient has reduced in metaphase. And the gradient of friction coefficient becomes steep, as shown in figure 19b. Figure 21 shows the statistical results of shift process at metaphase of wet clutch service life.

Figure 21a shows that the shift time increases with decrease of rising rate of clutch pressure, and the threshold of micro-slip has little effect on the shift time. Figure $21 \mathrm{~b}$ shows that, when the clutch pressure rises at fast or medium speed, the oscillations in the driveline becomes intense with the increase of threshold of micro-slip at first, and then, attenuates. When the pressure rises at slow speed, the oscillations in the driveline increase with the increase of threshold of micro-slip at first, and then dose not change with the variation of threshold of micro-slip. Figure 21c shows that friction loss increases with the increase of 
threshold of micro-slip, shift with slow rising speed of pressure and large threshold of micro-slip results in more frictional loss. Figure 21d shows that, the propensity of stick-slip phenomenon decreases as the threshold of micro-slip increases. Therefore, at metaphase of wet clutch life, slow or medium rising speed of pressure and medium threshold of micro-slip should be adopted to obtain good performance of shift.
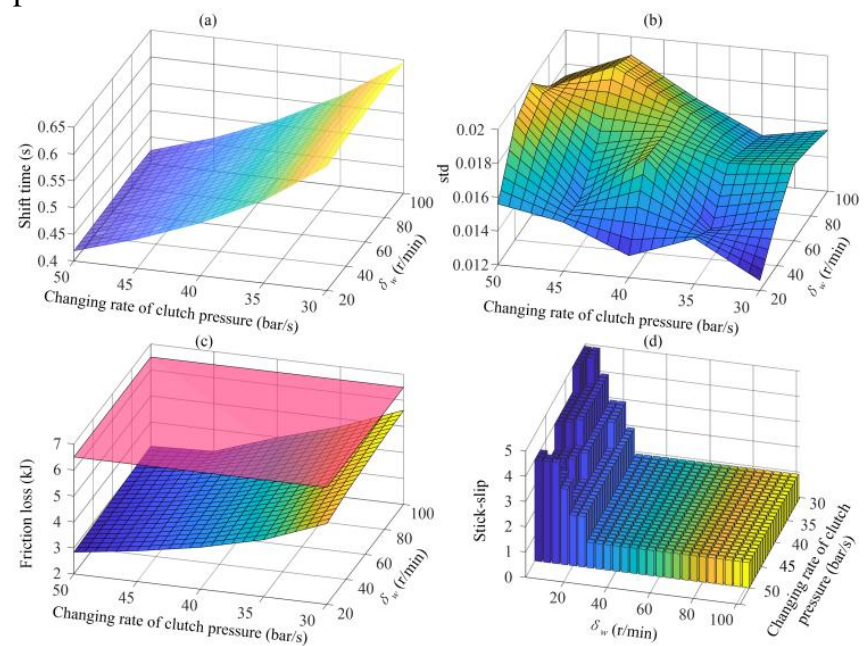

Figure 21 Coupling effect of controllable parameters on dynamic characteristics of driveline at metaphase

\section{Final stage}

When the clutch at at final stage, the friction characteristics of clutch becomes worse, the static friction coefficient is much lower than that at early stage and the gradient of friction coefficient is much steeper than that at early stage, as can be seen in figure $19 \mathrm{~b}$.
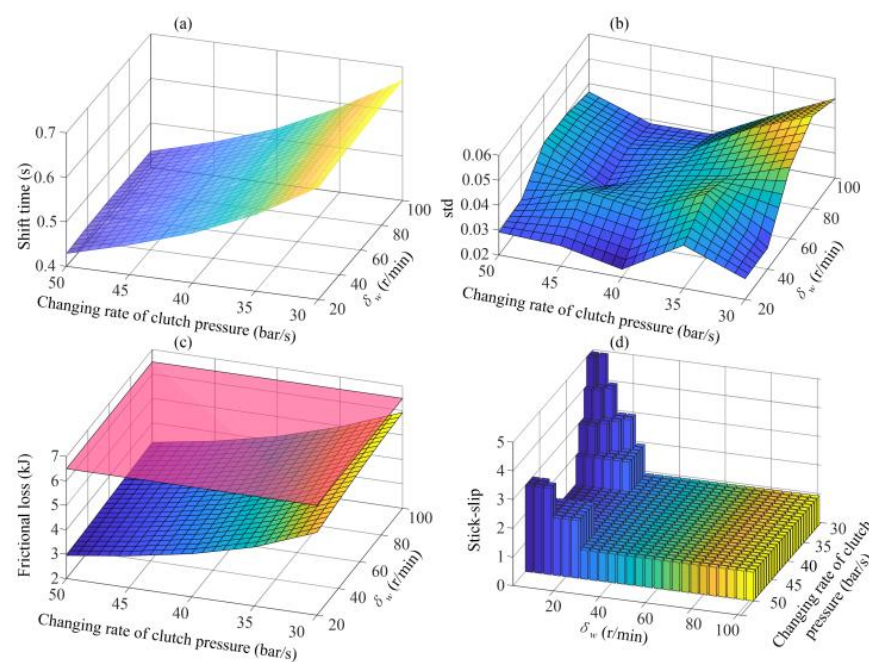

Figure 22 Coupling effects of controllable parameters on dynamic characteristics of driveline at final stage

Figure 22a shows that, at final stage of clutch service life, the shift time increases with decrease of rising rate of clutch pressure, and the threshold of micro-slip has little effect on the shift time. Figure $22 \mathrm{~b}$ shows that, when the pressure rises at a fast speed, the oscillation in the driveline becomes intense with the increase of the threshold of micro-slip. When the pressure rises at medium speed, the oscillation in the driveline becomes intense with the increase of the threshold of micro-slip at first, and then dose not change with the increase of the threshold of micro-slip. When the pressure rises at a slow speed, the oscillation in the driveline becomes intense with the increase of threshold of micro-slip. Figure 22c shows that friction loss increases with the increase of threshold of micro-slip, shift with slow rising speed of pressure and large threshold of micro-slip results in more frictional loss. Figure 22d shows that, the propensity of stick-slip phenomenon decreases as the threshold of micro-slip increases, and when rising the pressure at medium speed, the propensity of stick-slip phenomenon decreases. Therefore, at final stage of clutch service life, medium and fast speed of clutch pressure should be adopted to obtain a good performance of shift.

\section{Conclusions}

To investigate the dynamic characteristics of DCT during shift process along with the service life, an improved model was established with consideration of frictional characteristics of clutch, time-varying mesh stiffness and the torque coupling effect. The model was validated with the test data. Based on the model, the effect of frictional characteristics of clutch on the dynamic characteristics of driveline shift process was analyzed. To attenuate the oscillations in the driveline during shift process, a control strategy was proposed, which control the rising rate of on-coming clutch pressure and the maximum pressure coordinately. Since the friction characteristic of clutch varies with service life, the effect of these two controllable parameters on the dynamic characteristics of driveline at three different stages of clutch service life was investigate and following conclusions can be drawn.

(1) At early stage of clutch service life, the driveline suffers severe oscillation when the rising rate of pressure is fast, and applying slow rise speed of pressure and medium threshold of micro-slip can obtain good performance of shift.

(2) At metaphase of clutch service life, the influence law is complicated. When the clutch pressure rises at fast or medium speed, the oscillations in the driveline becomes intense with the increase of threshold of 
micro-slip at first, and then, attenuates. When the pressure rises at slow speed, the oscillations in the driveline increase with the increase of threshold of micro-slip at first, and then dose not change with the variation of threshold of micro-slip. When the pressure rises at medium speed, the propensity of stick-slip phenomenon is smaller than that at slow of fast speed. And applying medium rise speed of clutch pressure and medium threshold of micro-slip can obtain good performance of shift.

(3) The driveline suffers severe oscillations at final stage of clutch service life since the degradation of friction characteristics. However, with combination of medium or fast rise rate of pressure and medium threshold of micro-slip, relatively good performance of shift can also be attained.

\section{Declaration}

\section{Acknowledgements}

The authors sincerely thanks to Professor Datong Qin of Chongqing University for his critical discussion and reading during manuscript preparation.

\section{Funding}

Supported by National Key Research and Development Project (Grant No. 2016YFD0701102), National Natural Science Foundation of China (Grant No. U1764259), Fundamental Research Funds for the Central University (Grant No. 2018CDJDCD0001)

\section{Availability of data and materials}

The datasets supporting the conclusions of this article are included within the article.

\section{Authors' contributions}

The author' contributions are as follows: Dongyang Wang was in charge of the whole trial; Dongyang Wang wrote the manuscript; Minghui $\mathrm{Hu}$ assisted with sampling and laboratory analyses; Datong Qin assisted with providing critical comments on the manuscript.

\section{Competing interests}

The authors declare no competing financial interests.

\section{Consent for publication}

Not applicable

\section{Ethics approval and consent to participate}

Not applicable

\section{References}

[1] M. Adhitya, R. Mustafa, A .Plötner, et al., A new control strategy of wet dual clutch transmission (DCT) clutch and synchronizer for seamless gear preselect, J. SAE Int. J. Passeng. Cars-Mech. Syst. 6 2013, (2013-01-0340) 535-551.

[2] Kim S, Oh J, Choi S. Gear shift control of a dual-clutch transmission using optimal control allocation. Mechanism and Machine Theory, 2017, 113: 109-125.

[3] Petrescu R V, Aversa R, Apicella A, et al. Dynamic Synthesis of a Dual-Clutch Automatic Gearboxes. American Journal of Engineering and Applied Sciences, 2018, 11(2): 663-679.

[4] Kim S, Choi S. Control-oriented modeling and torque estimations for vehicle driveline with dual-clutch transmission. Mechanism and Machine Theory, 2018, 121: 633-649.

[5] Lei Z, Sun D, Liu Y, et al. Analysis and coordinated control of mode transition and shifting for a full hybrid electric vehicle based on dual clutch transmissions. Mechanism and Machine Theory, 2017, 114: 125-140.

[6] Cho J, Lee Y, Kim W, et al. Wet single clutch engagement behaviors in the dual-clutch transmission system. International Journal of Automotive Technology, 2018, 19(3): 463-472.

[7] E. Galvagno, M. Velardocchia, A. Vigliani, Dynamic and kinematic model of a dual clutch transmission, J. Mech. Mach. Theory. 2011, 46 (6): 794-805.

[8] P. D. Walker, A. S. Rahman, B Zhu, et al., Modelling simulations and optimisation of electric vehicles for analysis of transmission ratio selection, J. Adv. Mech. Eng. 2013, 5, 340-435.

[9] P. D Walker, N. Zhang, R. Tamba, Control of gear shifts in dual clutch transmission powertrains, J. Mech. Syst. Signal Proc. 2010, 25 (6): 1923-1936.

[10]P. D. Walker, N. Zhang, W. Zhan, et al., Modelling and simulation of gear synchronisation and shifting in dual-clutch transmission equipped powertrains, J. Proc. Inst. Mech. Eng. Part C-J. Eng. Mech. Eng. Sci., 2013, 227 (2): 276-287.

[11]M Goetz, M Levesley, D Crolla, Dynamic modelling of a twin clutch transmission for controller design, Materials Science Forum, 2003, Vols. 440-441, 253-260,.

[12]M Goetz, M Levesley, D Crolla, Dynamics and control of gearshifts on twin-clutch transmissions, J. Proc. Inst. Mech. Eng. Part D-J. Automob. Eng. 2005, 219 (8): 951-963.

[13]M Kulkarni, S Thim, Y Zhang, Shift dynamics and control of dual-clutch transmissions, J. Mech. Mach. Theory. 2007, 42 (2): 168-182.

[14]Y Hu, L Tian, B Gao, et al., Nonlinear gearshifts control of dual-clutch transmissions during inertia phase, J. ISA Trans. 2014, 53 (4): 1320-1331.

[15]J.OH Jiwon, B.Choi Seibum, Real-time estimation of transmitted torque on each clutch for ground vehicles with dual clutch transmission, J. IEEE-ASME Trans. Mechatron. 2015, 20 (1) :24-36.

[16]J.OH Jiwon, B.Choi Seibum, J Kim. Driveline modeling and estimation of individual clutch torque during gear shifts for dual clutch transmission, J. Mechatronics. 2014, 24 (5): 449-463.

[17]P.D.Walker, N. Zhang, Numerical investigations into shift transients of a dual clutch transmission equipped powertrains with multiple nonlinearities, J. J Vib Control. 2015, 21 (8): 1473-1486.

[18]W. B. Yang, G.-Q. Wu, D.-T. Qin. Drive line system modeling and shift characteristic of dual clutch transmission powertrain, J. CJME. 2007, 43 (7): 188-194. 
[19]Z. G. Zhao, X.-T. Hu, J.-L. Jiang, et al., T. Zhang Slip mode variable structure coordinating control and real-time optimization of launch for dry dual clutch transmission, J. CJME , 2012, 48 (24): 87-105.

[20]Z. G. Zhao, Q. Wang, H.-J. Chen, et al., Torque coordinating optimal control for dry dual clutch transmission in shifting process [J]. J Automotive Safety and Energy, 2013, 4(3): 279-278.

[21]Y. Liu, D. Qin, H. Jiang, et al., A systematic model for dynamics and control of dual clutch transmissions, J. J. Mech. Des, 2009, 131 (6) 061012.

[22]X Xu, X Han, Y Liu, et al., Modeling and Dynamic Analysis on the Direct Operating Solenoid Valve for Improving the Performance of the Shifting Control System, J. Appl. Sci., 2017, 7 (12): 1266.

[23]D. G. Lewicki, H. Desmidt, E. C. Smith, et al., Dynamics of a dual-clutch gearbox system: analysis and experimental validation, $J$. J. Am. Helicopter Soc. , 2013, 58 (1): 17-28.

[24]B. Mashadi., D. Crolla. Vehicle Powertrain System. John Wiley \& Sons, 2012.

[25]Berglund K, Marklund P, Larsson R. Lubricant ageing effects on the friction characteristics of wet clutches. Proceedings of the Institution of Mechanical Engineers, Part J: Journal of Engineering Tribology, 2010, 224(7): 639-647.

[26]Stembalski M, Preś P, Skoczynski W, et al. Determination of the friction coefficient as a function of sliding speed and normal pressure for steel C45 and steel 40HM. ACME, 2013, 13: 444-448.

[27]S. Iqbal, F. Al-Bender, A. P. Ompusunggu, B. Pluymers, et al., Modeling and analysis of wet friction clutch engagement dynamics, Mech. Syst. Signal Process., 2015, 60: 420-436.

[28]D. Centea, H. Rahnejat, M. Menday, Non-linear multi-body dynamic analysis for the study of clutch torsional vibrations (judder), Appl. Math. Model., 2001, 25 (3): 177-192.

[29]D. Wang, M. Hu, B. Li, D. Qin and D. Sun, "Study on the Influence Factors Upon the Propensity to Stick-Slip Phenomenon During Vehicle Start-Up Process," in IEEE Access, vol. 8, pp. 12343-12353, 2020, doi: 10.1109/ACCESS.2020.2966001.

[30]C. Duan, R. Singh, "Stick-slip behavior of torque converter clutch", Aug. 2010.

[31] Y. Yang, Li, M., Hu, M, et al. Influence of controllable parameters on load sharing behavior of torque coupling gear set. Mechanism and Machine Theory, 2018, 121: 286-298.

[32]Zhou B, Lu T, Zhang J, et al. Micro-slip control and torque estimation for dual clutch transmission. Proceedings of the Institution of Mechanical Engineers, Part K: Journal of Multi-body
Dynamics, 2017, 231(4): 750-764.

[33]Lu T, Zhou B, Zhang J, et al. Clutch micro-slip control in gearshifts for dual clutch transmission. Proceedings of the Institution of Mechanical Engineers, Part K: Journal of Multi-body Dynamics, 2019, 233(1): 43-59.

[34]Fu Y, Liu Y, Cui L, et al. Dynamic analysis and control strategy of wet clutches during torque phase of gear shift. Journal of Mechanical Science and Technology, 2016, 30(4): 1479-1496.

[35]Canale M, Cerone V, Baliva S, et al. A model predictive control approach to micro-slip control of automated manual transmission systems[C]//2017 American Control Conference (ACC). IEEE, 2017: 3613-3618.

\section{Biographical notes}

Dong-Yang Wang, born in 1990, is currently a $\mathrm{PhD}$ candidate at School of Automotive Engineering, Chongqing University, China. He received his bachelor degree from Henan University of Science and Technology, China, in 2014. His research interests include Vehicle driveline system and control.

E-mail:wdyang@cqu.edu.cn

Ming-Hui Hu, born in 1975, is currently a professor at Chongqing University, China. He received his Ph.D. degree on mechanical design in Chongqing University, China, in 2007.

Tel: +86-15826089252; E-mail: hu_ming @cqu.edu.cn

Da-Tong Qin, born in 1956, is currently a professor at State Key Laboratory of Mechanical Transmissions, Chongqing University, China.

E-mail: dtqin@cqu.edu.cn

\section{Appendix}

Appendix and supplement both mean material added at the end of a book. An appendix gives useful additional information, but even without it the rest of the book is complete: In the appendix are forty detailed charts. A supplement, bound in the book or published separately, is given for comparison, as an enhancement, to provide corrections, to present later information, and the like: A yearly supplement is issue. 
Figures
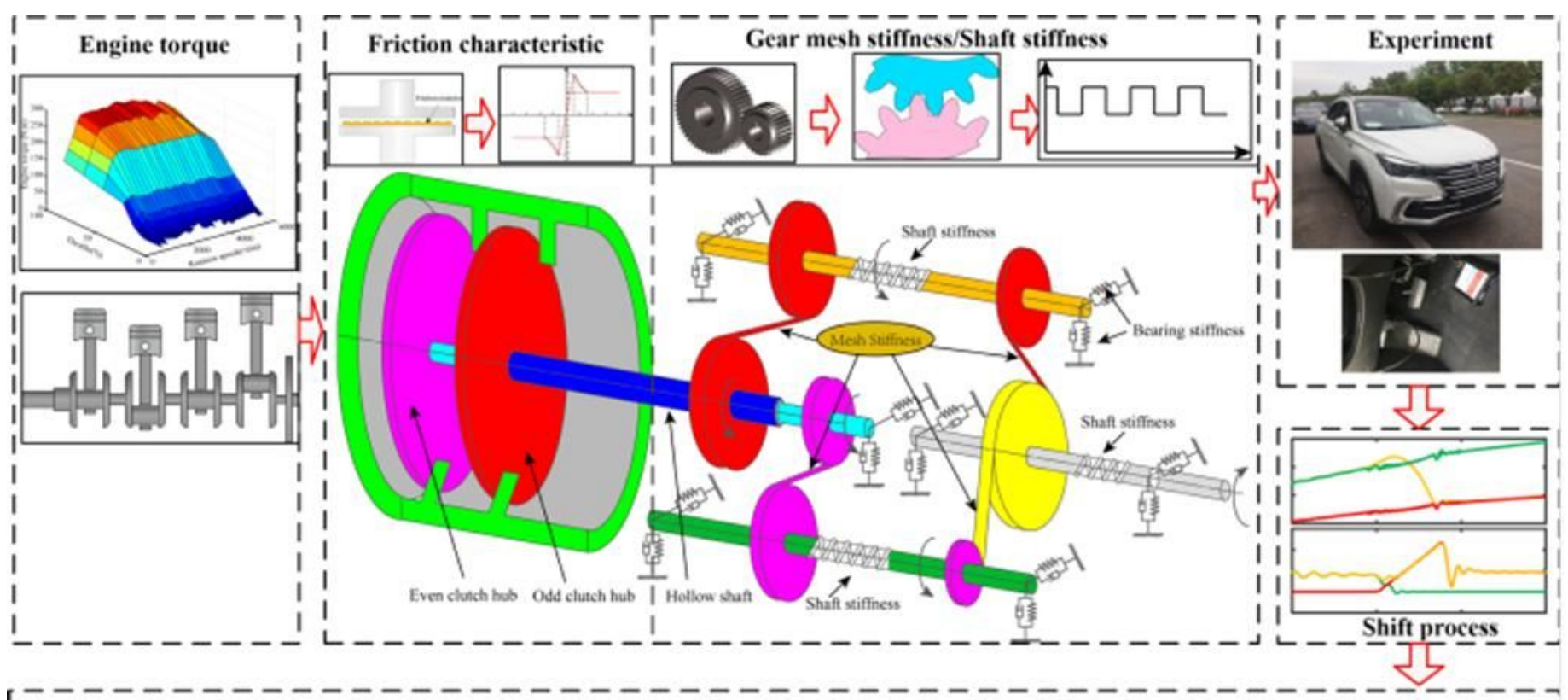

Effect of controllable parameters on dynamic characteristics of driveline during shift process throughout service life cycle
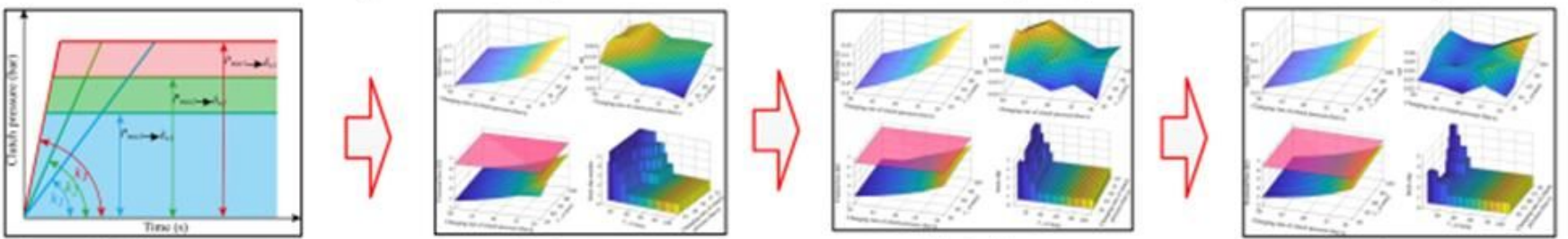

Controllable parameter

Early stage

Metaphase

Final stage

Figure 1

Structure of the paper 


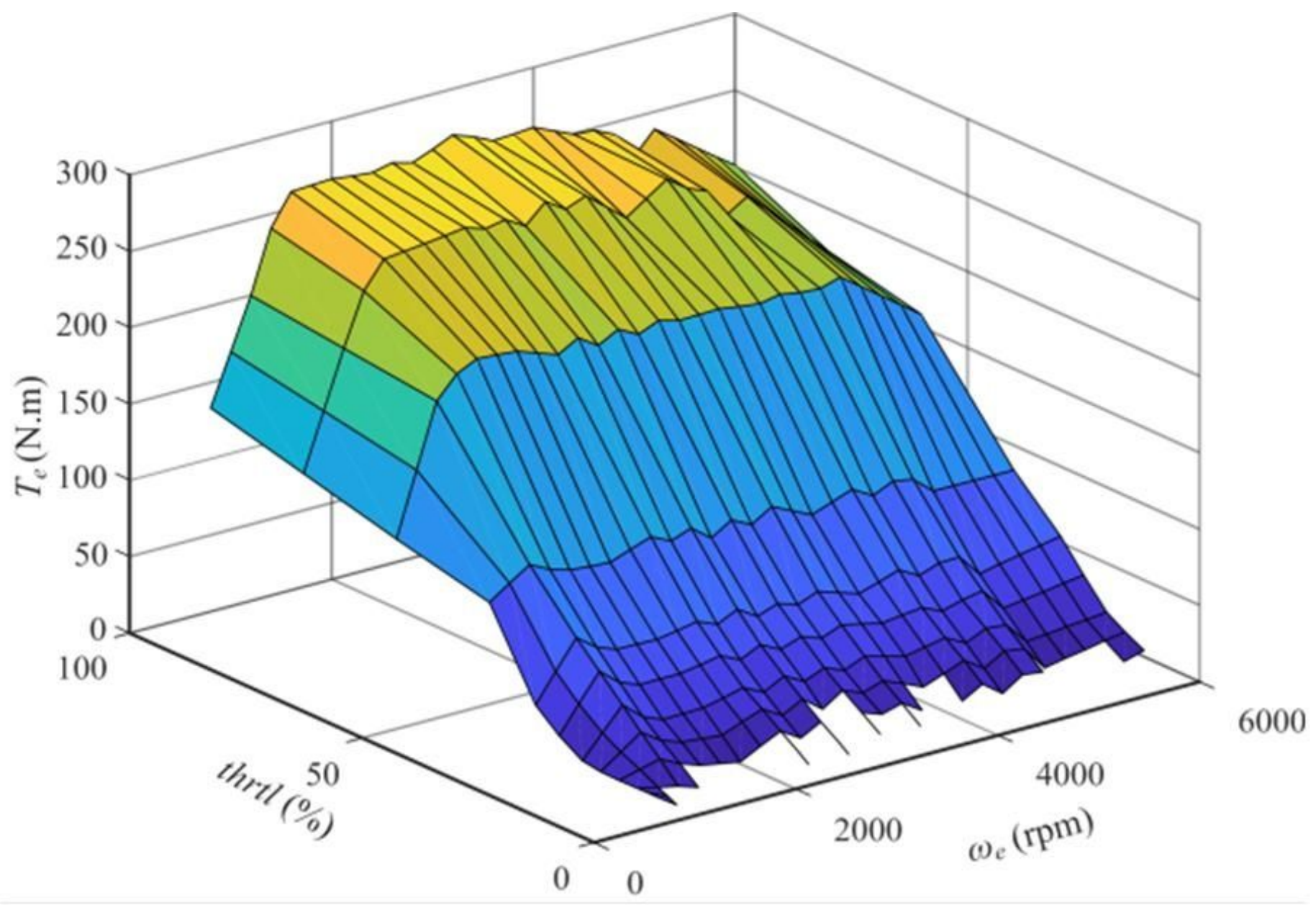

Figure 2

Map of the Engine 

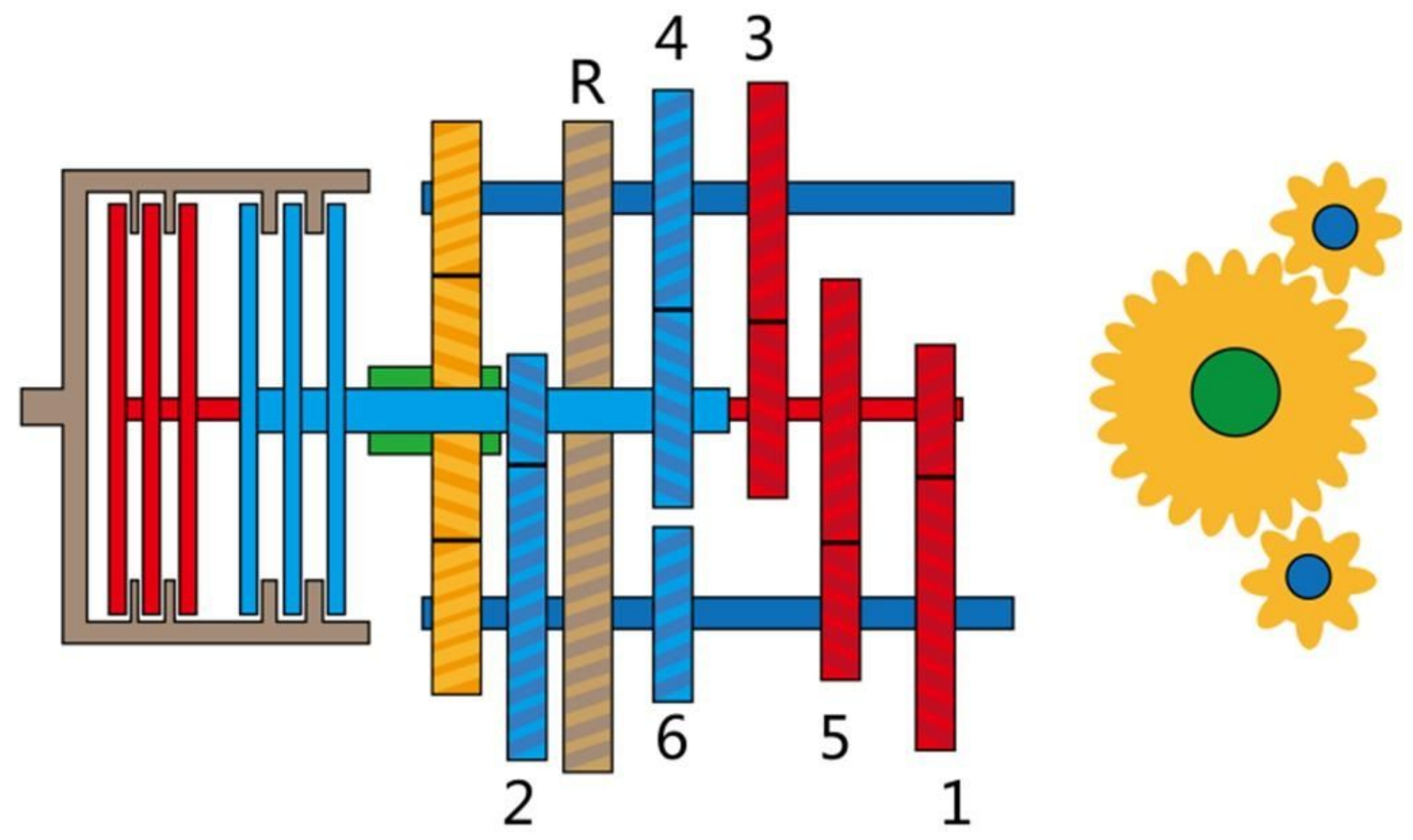

Figure 3

Schematic of DCT

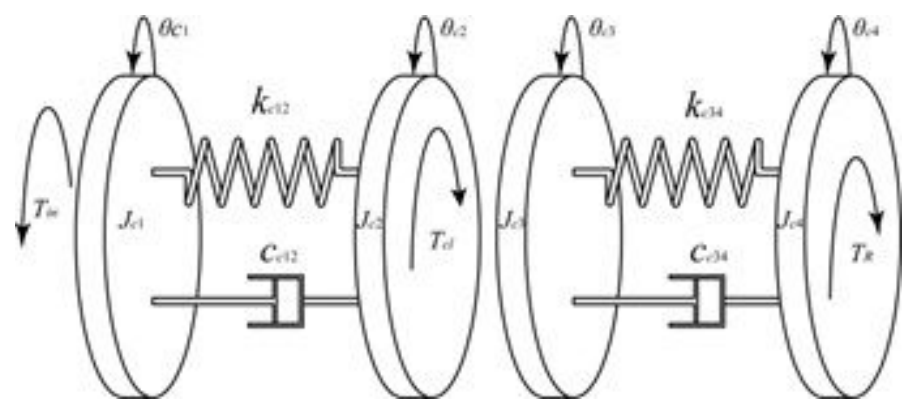

Figure 4

4 DOF model of the clutch 

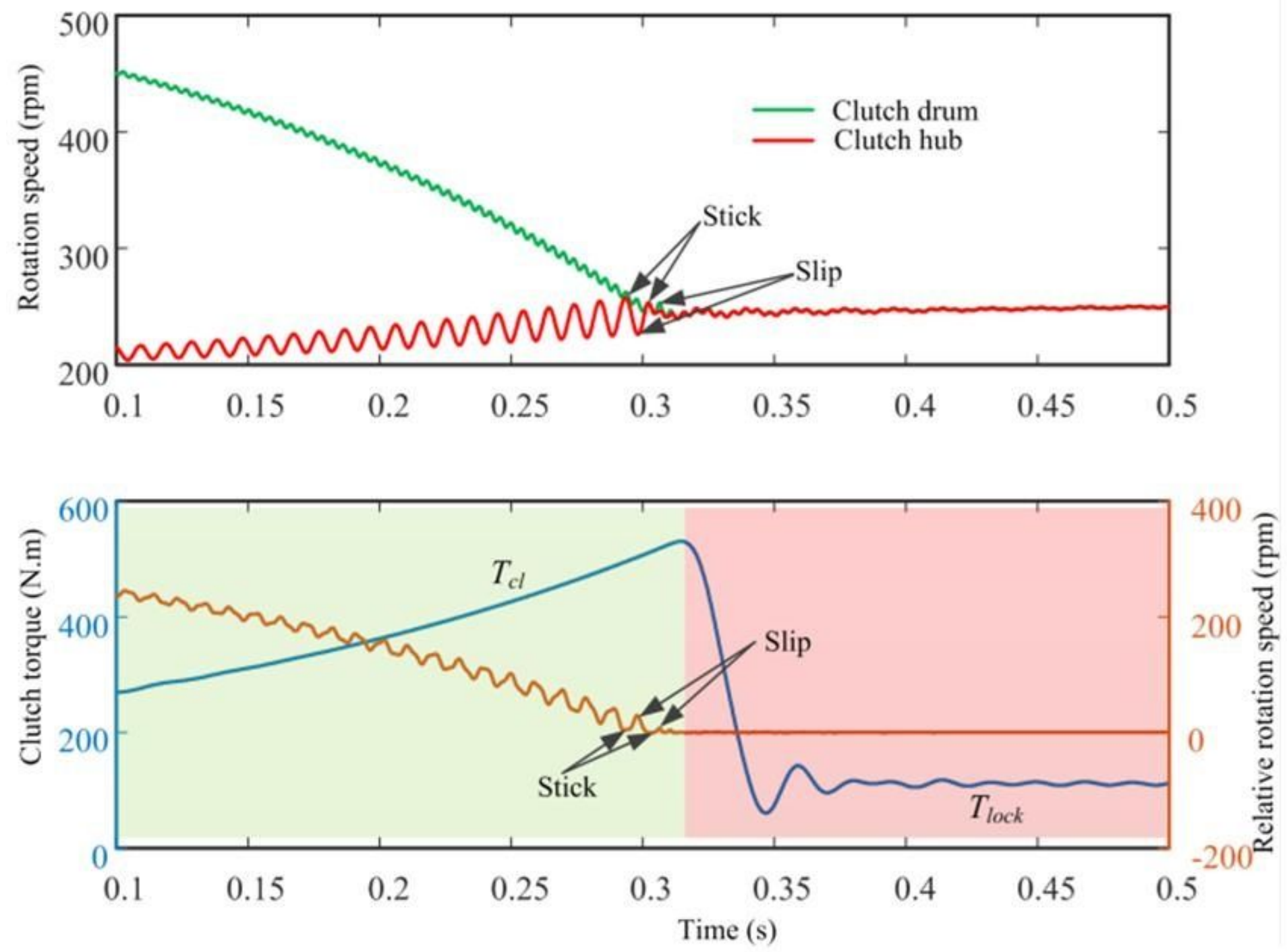

Figure 5

Dynamic characteristics of engaging clutch
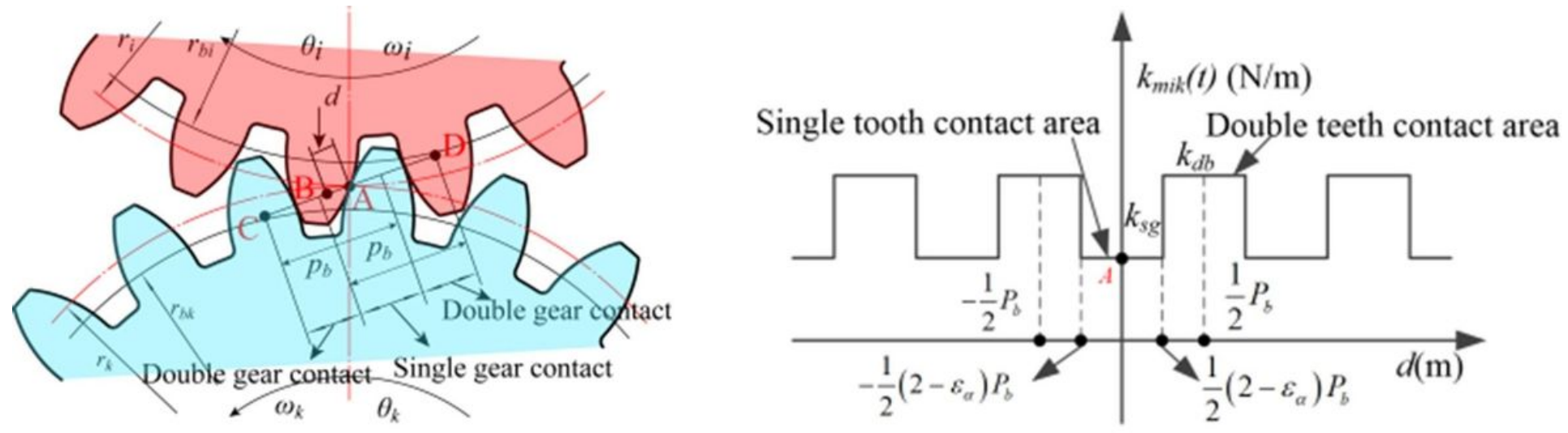

Figure 6

Time-varying mesh stiffness of gear set 


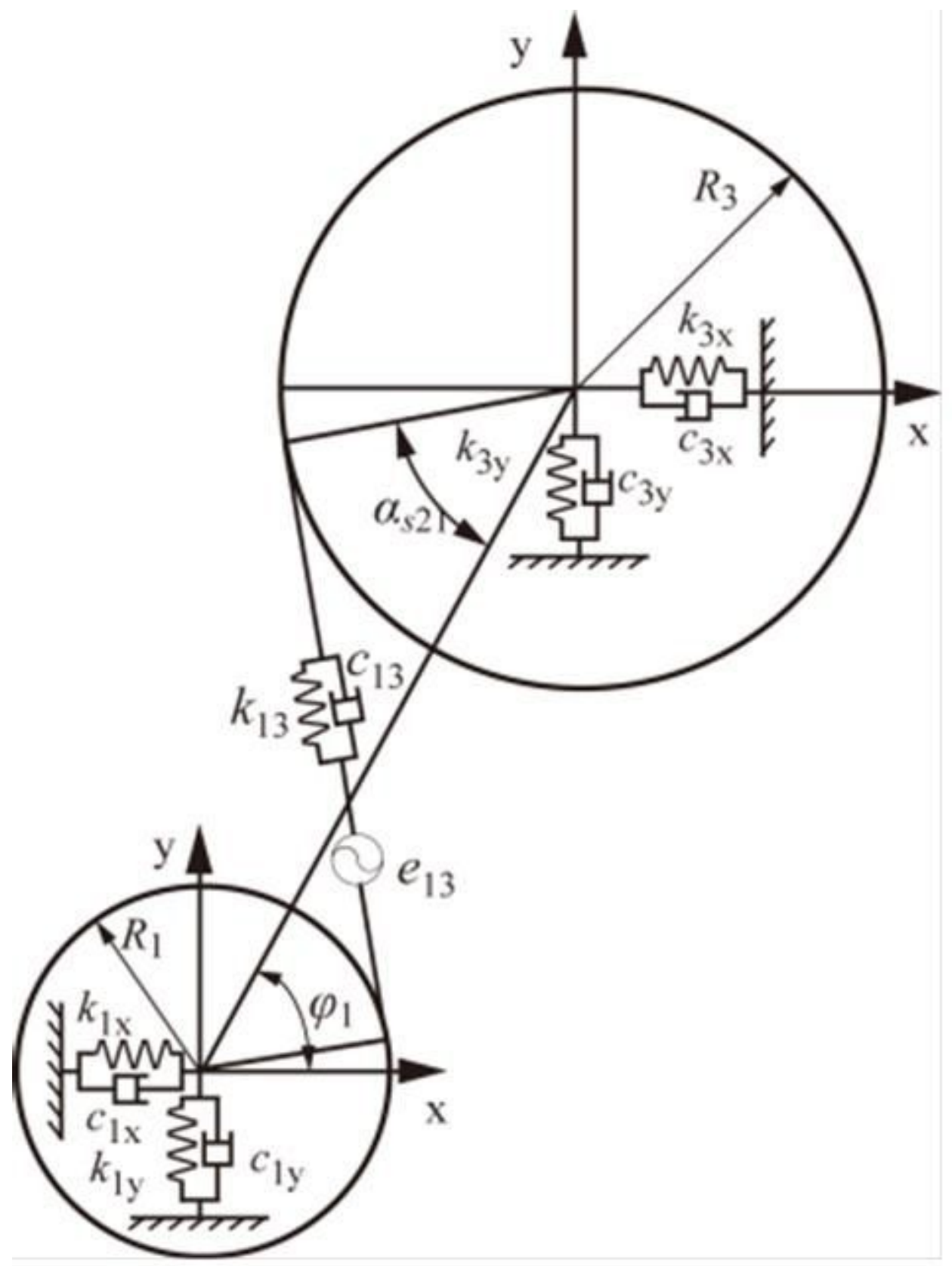

Figure 7

Model of a pair of meshing gears 


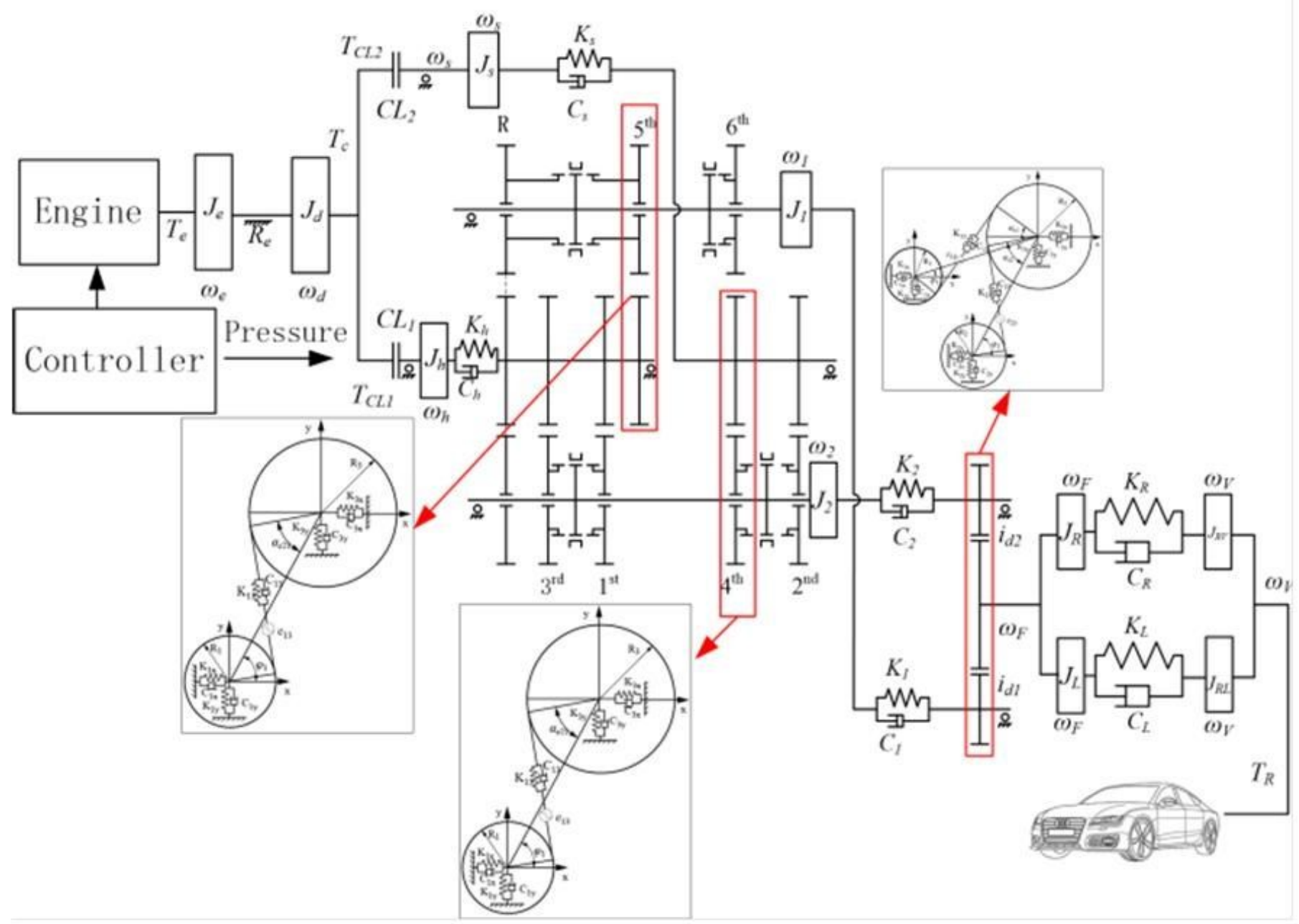

Figure 8

Dynamic model of gear system

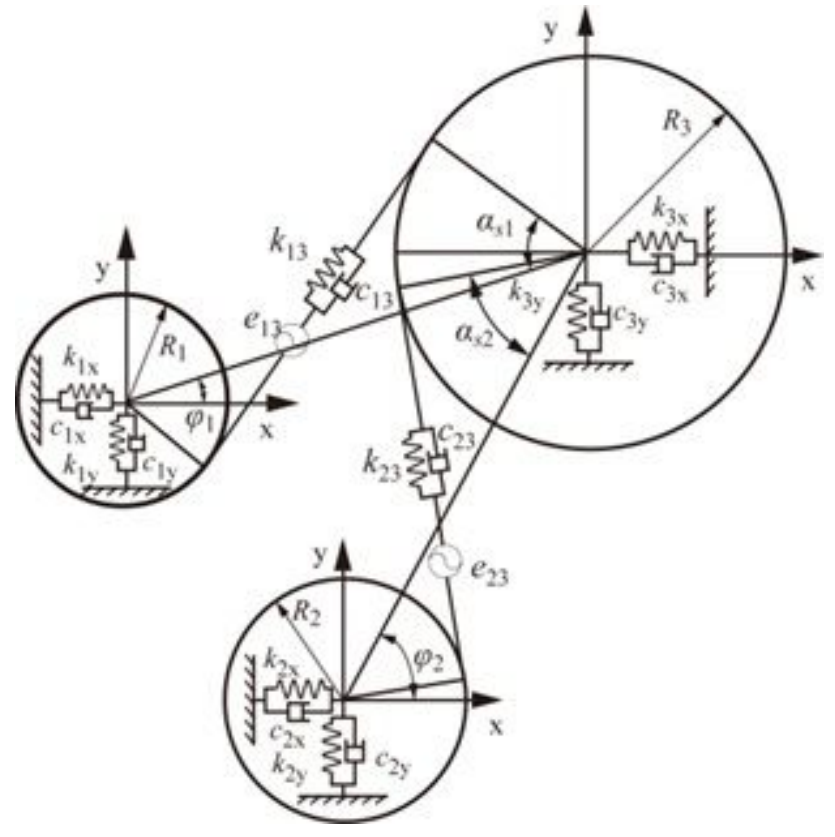

Figure 9 
Schematic of the vehicle powertrain system

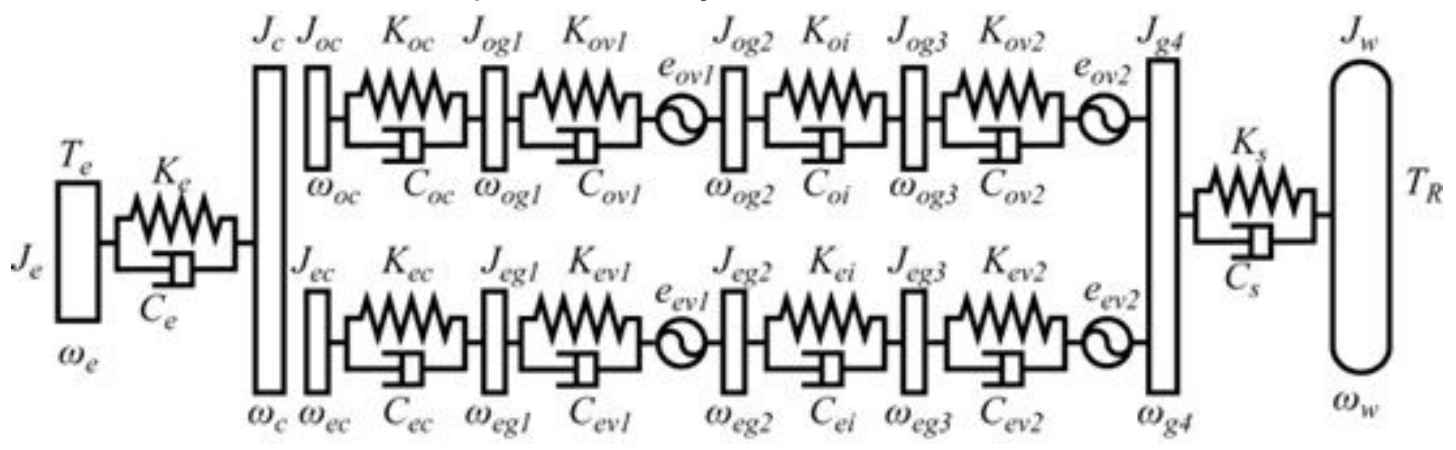

Figure 10

Dynamic model of vehicle powertrain system

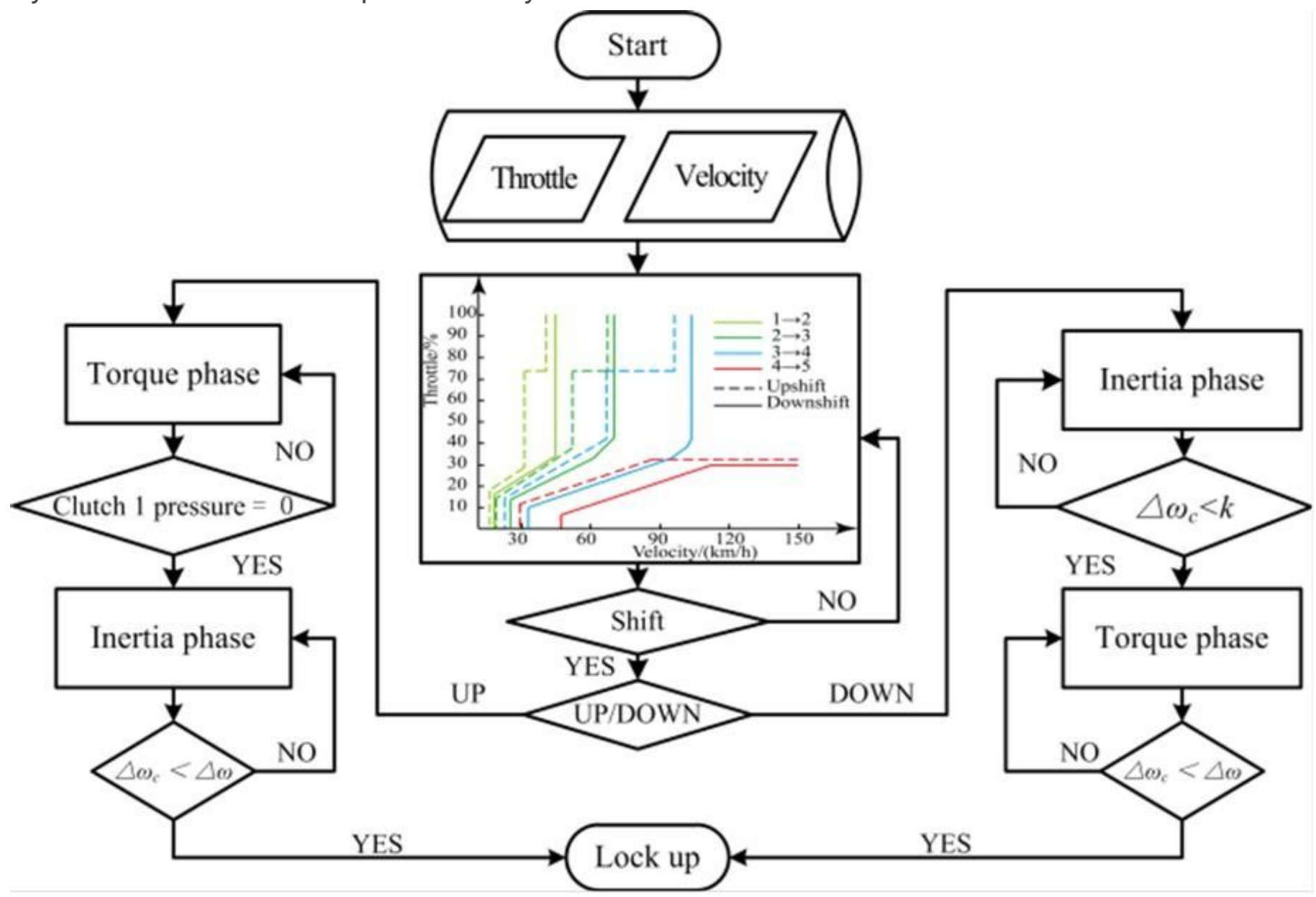

Figure 11

Gearshift logic 

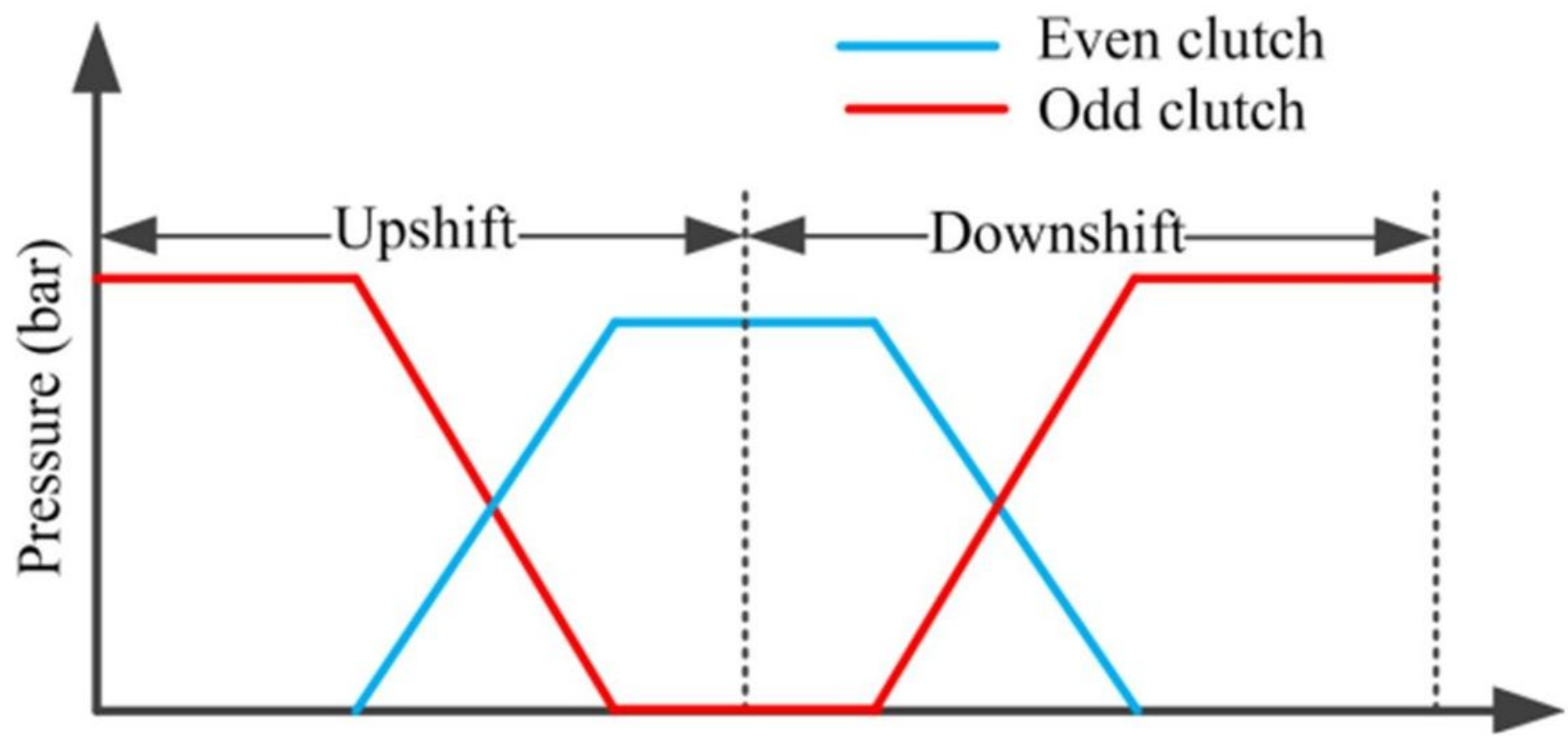

Figure 12

Clutch pressure control profile during shifting 
(a)

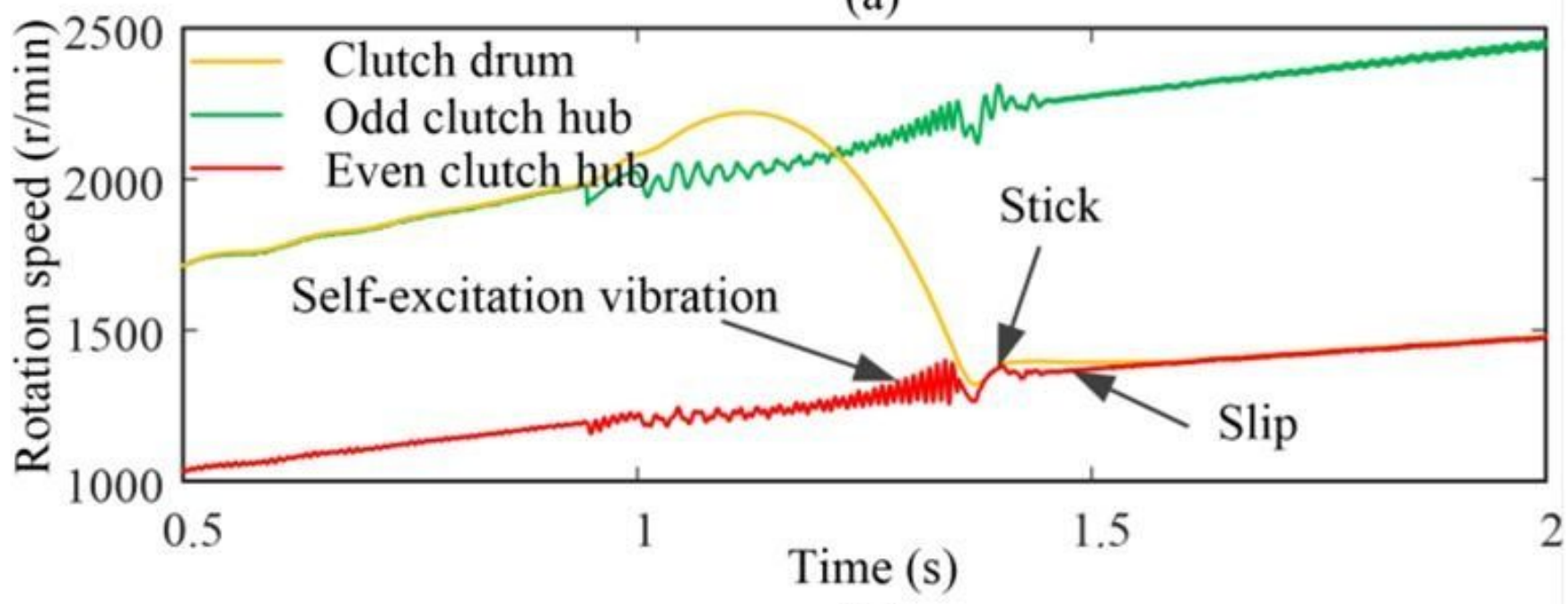

(b)

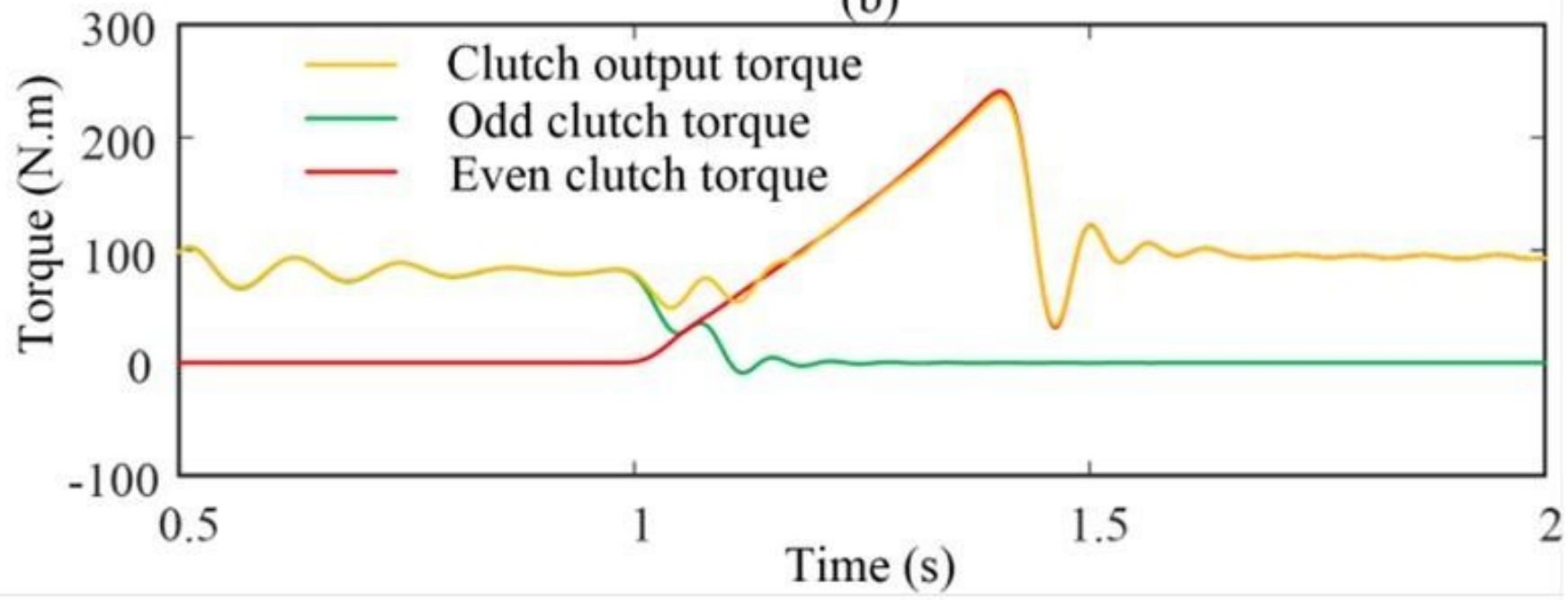

Figure 13

Simulation result of shift

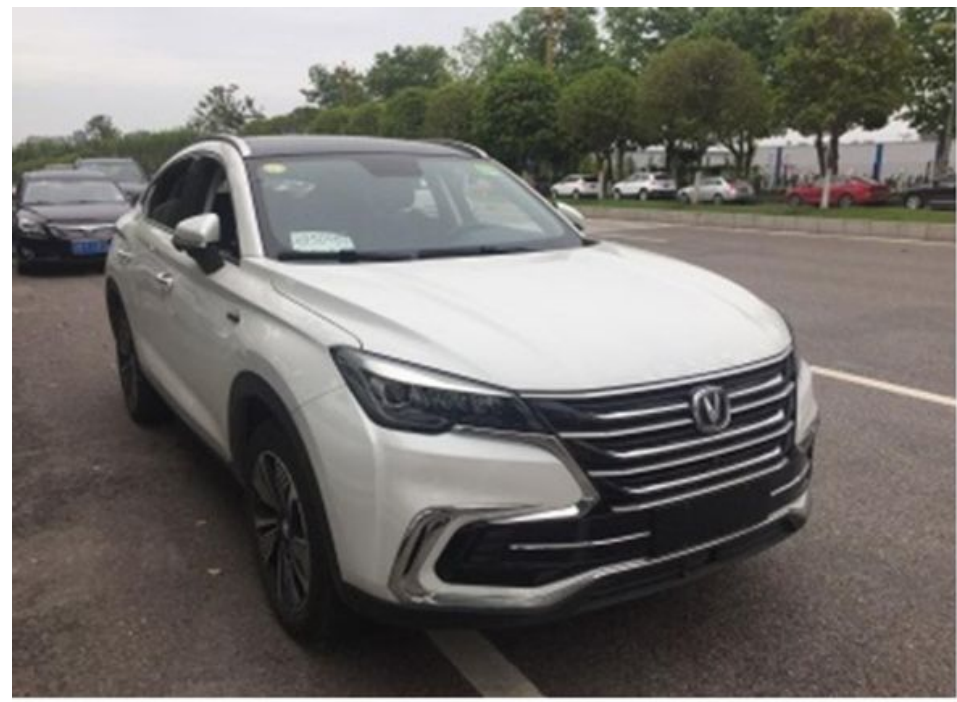

(a)

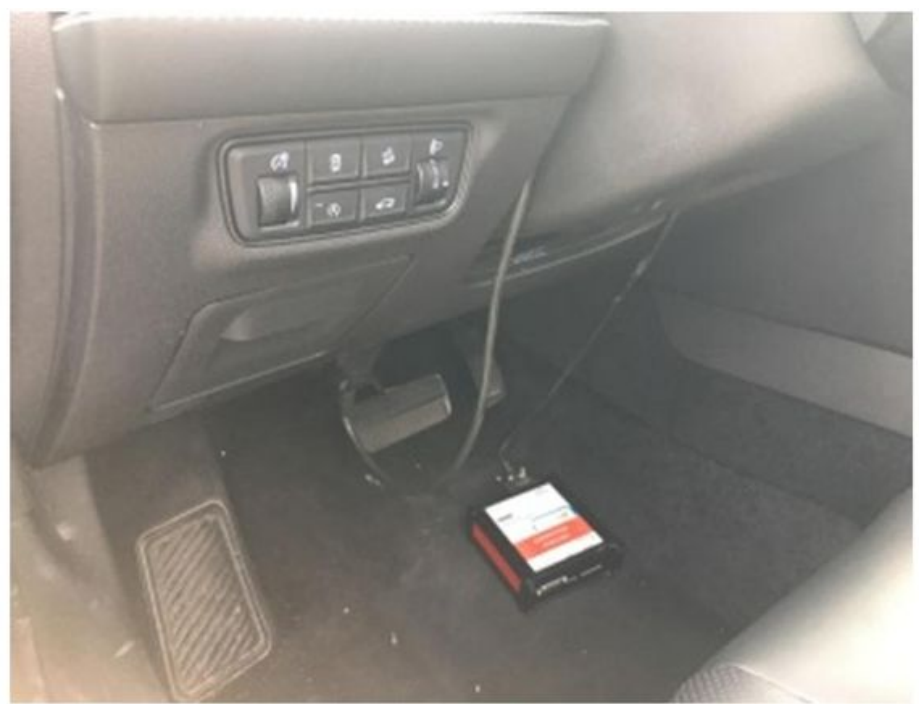

(b) 
Figure 14

Tested vehicle and data recorder

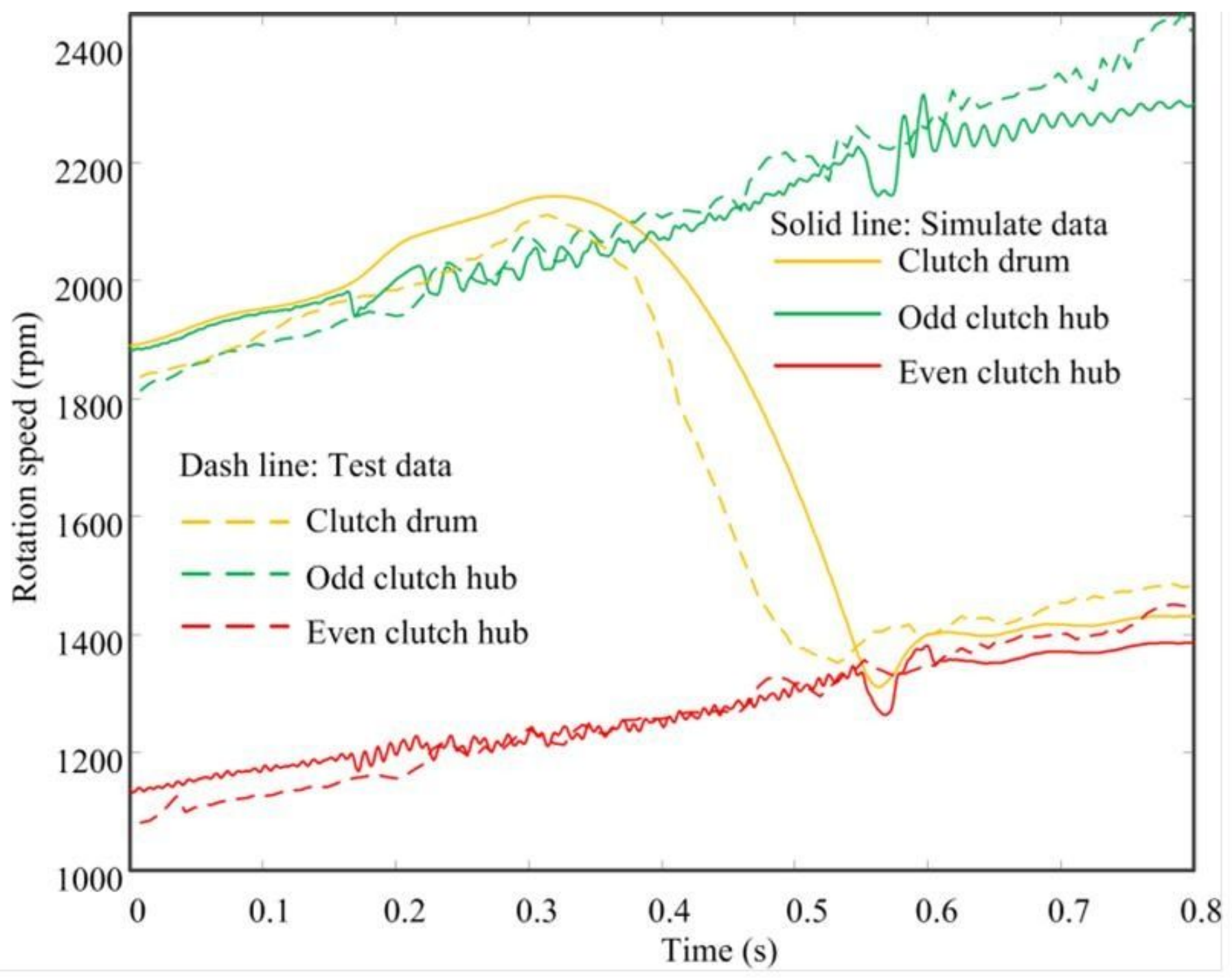

Figure 15

Comparison between the simulation and test 


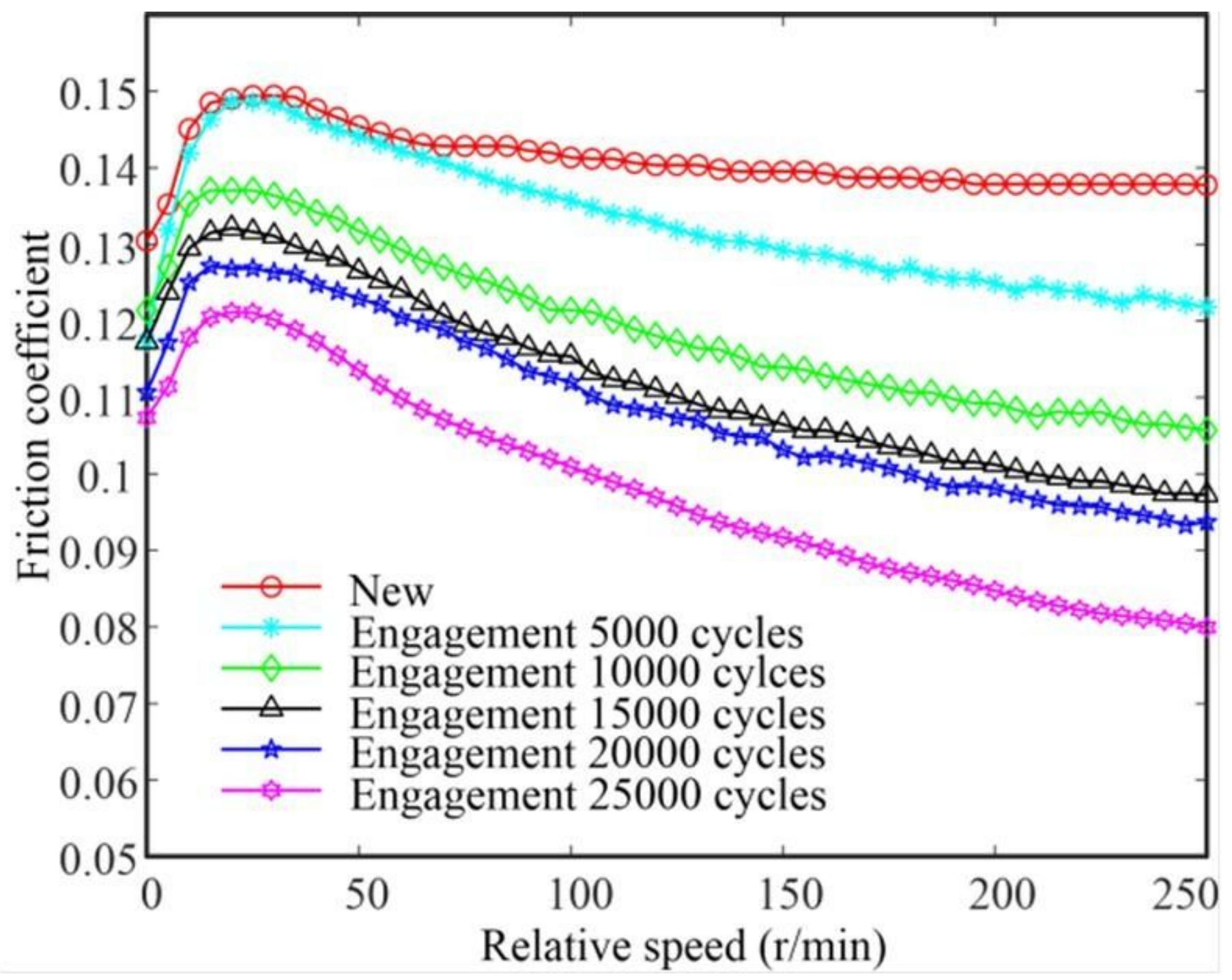

Figure 16

Friction coefficient of wet clutch at different stages 
(a)
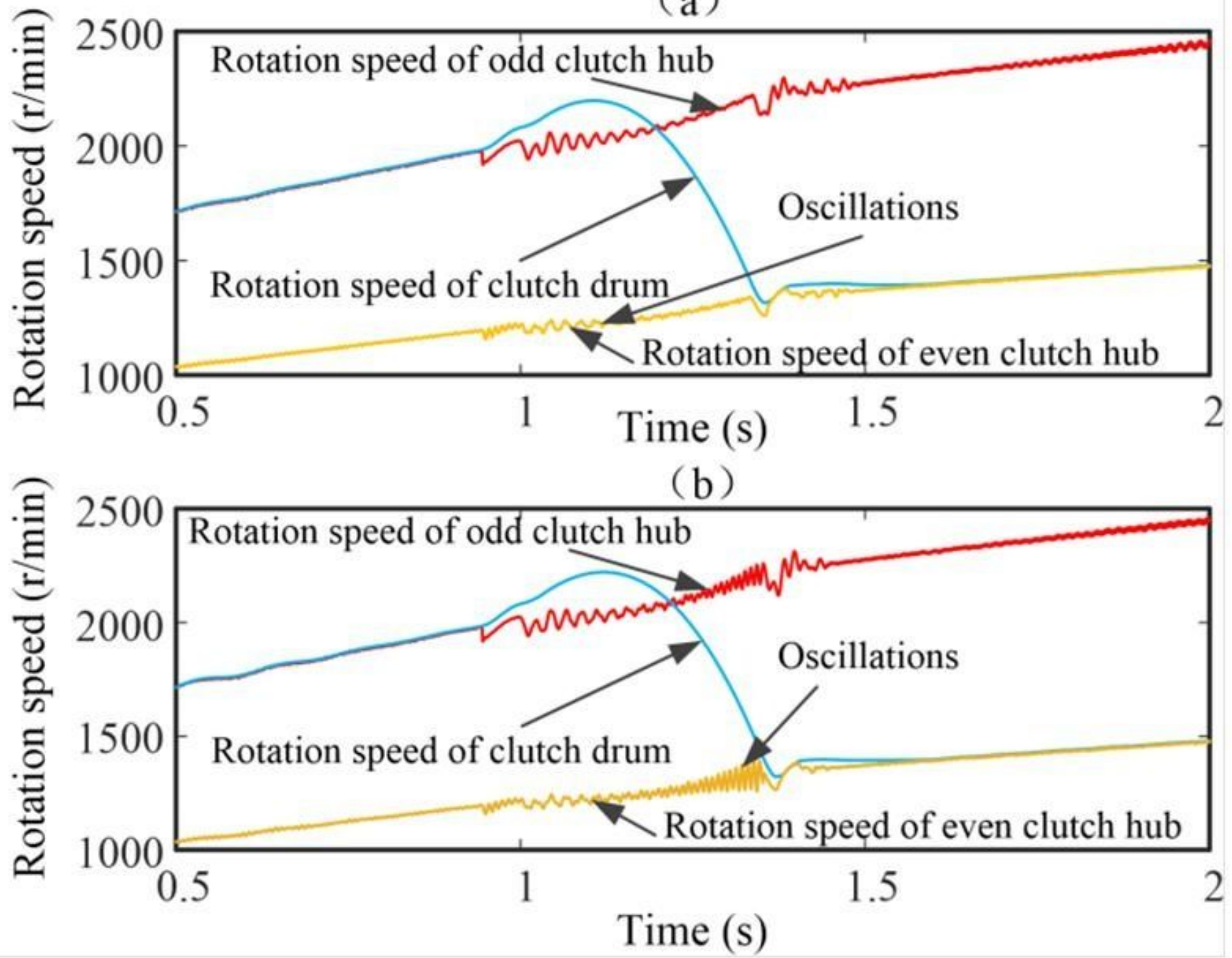

Figure 17

Effect of negative gradient of friction coefficient on dynamic characteristics of driveline 


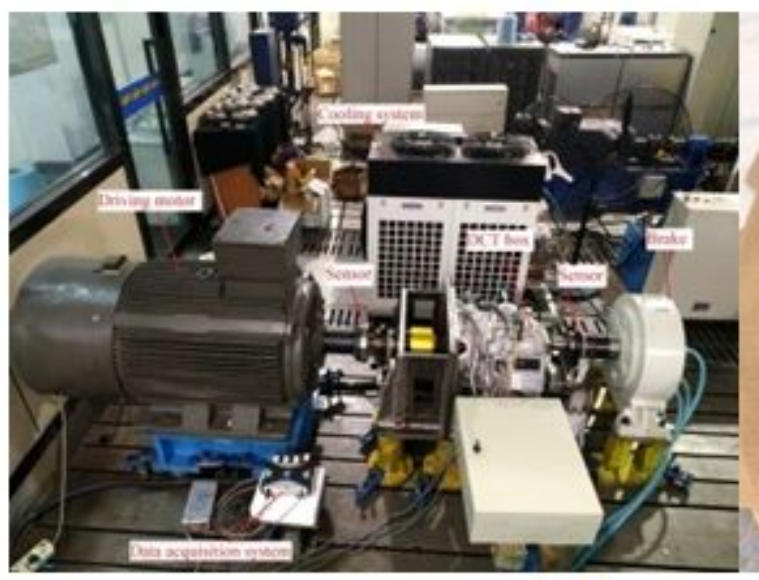

(a)

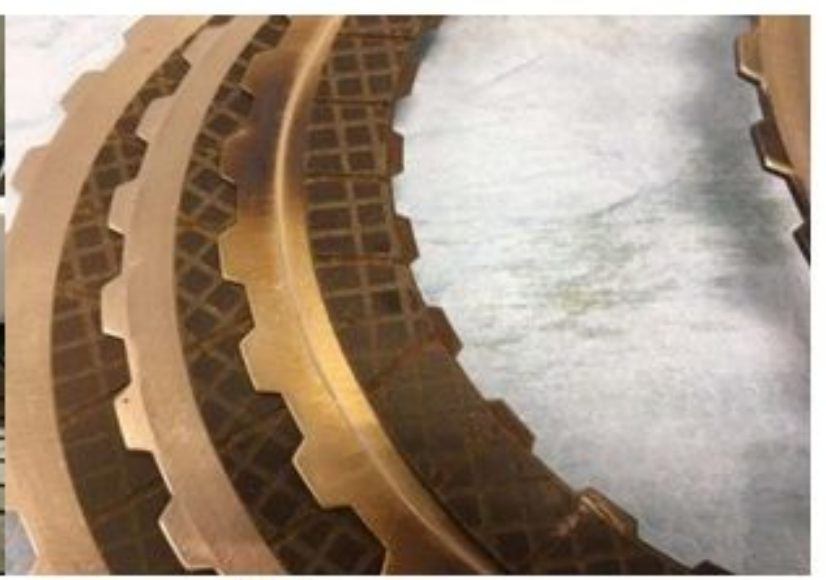

(b)

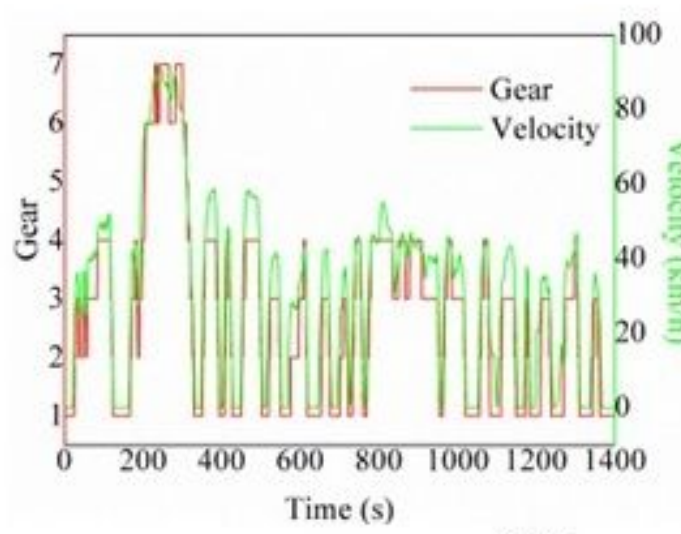

(c)

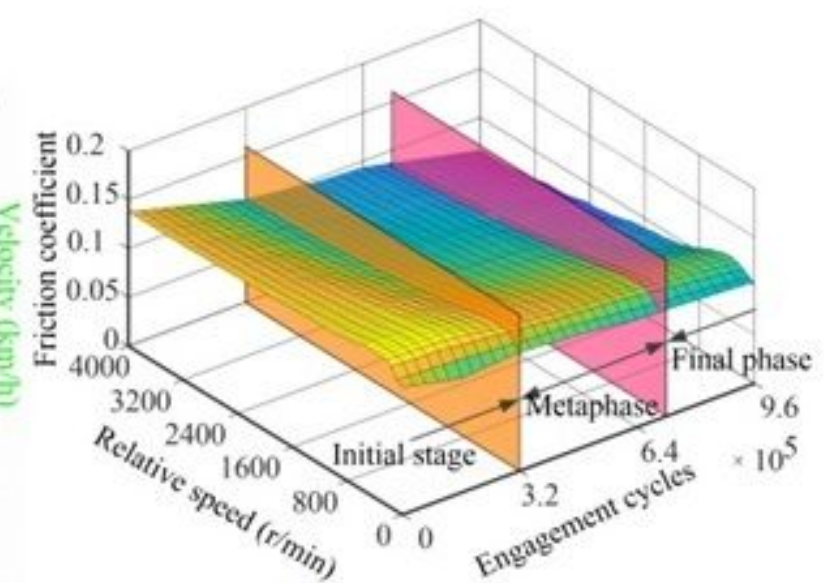

(d)

\section{Figure 18}

(a) Clutch friction plate; (b) test rig; (c) UDDS cycles and gear states of vehicle; (d) Division of the service life stages of wet clutch

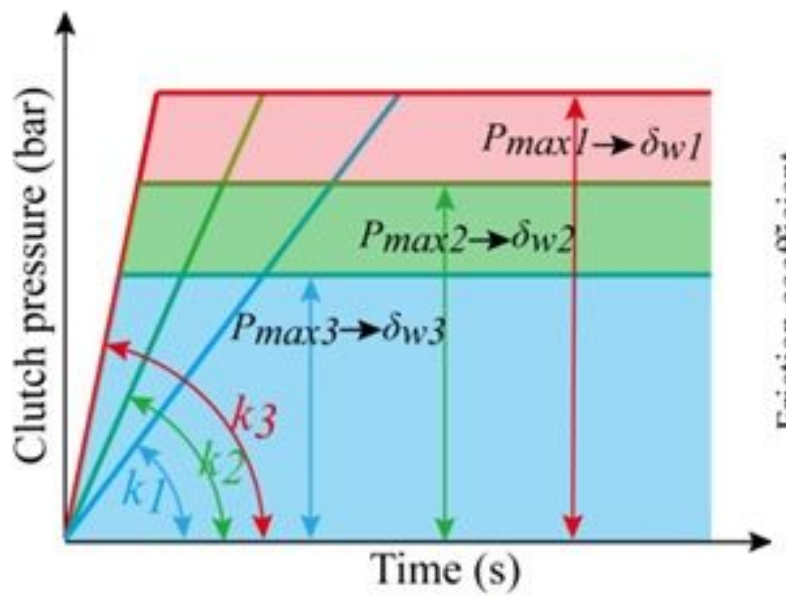

(a)

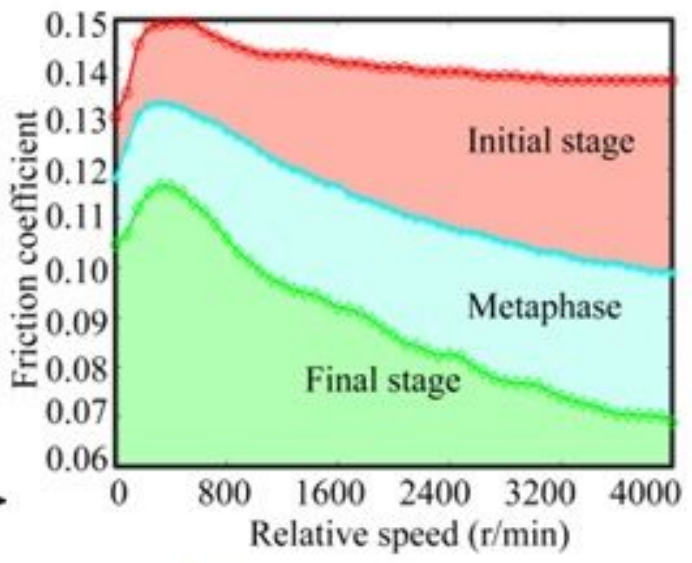

(b) 
Figure 19

(a) Rising rate of hydraulic pressure and maximum pressure; (b) friction coefficient at different stages

(a)

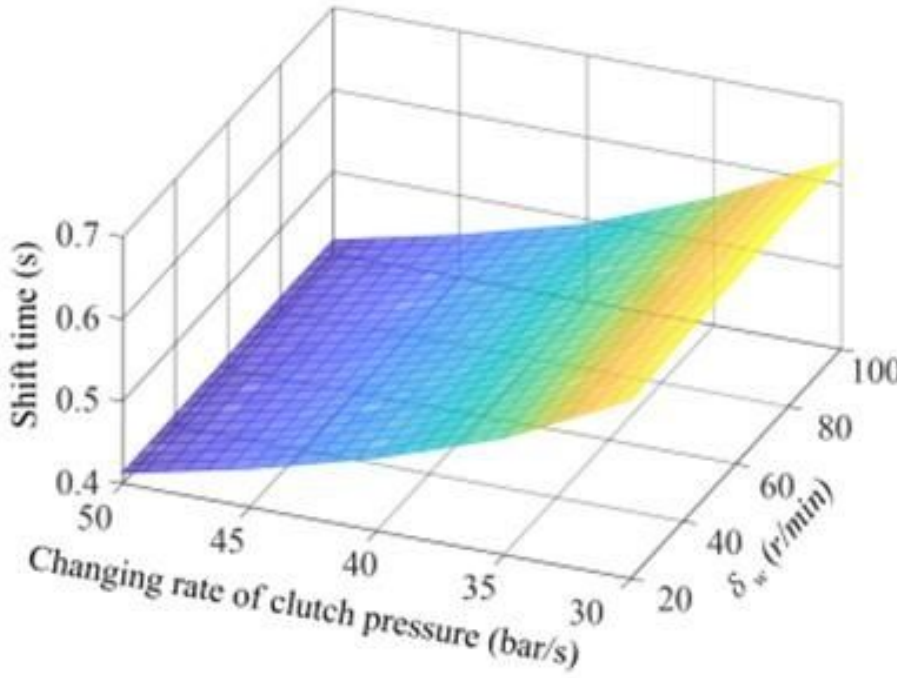

(c)

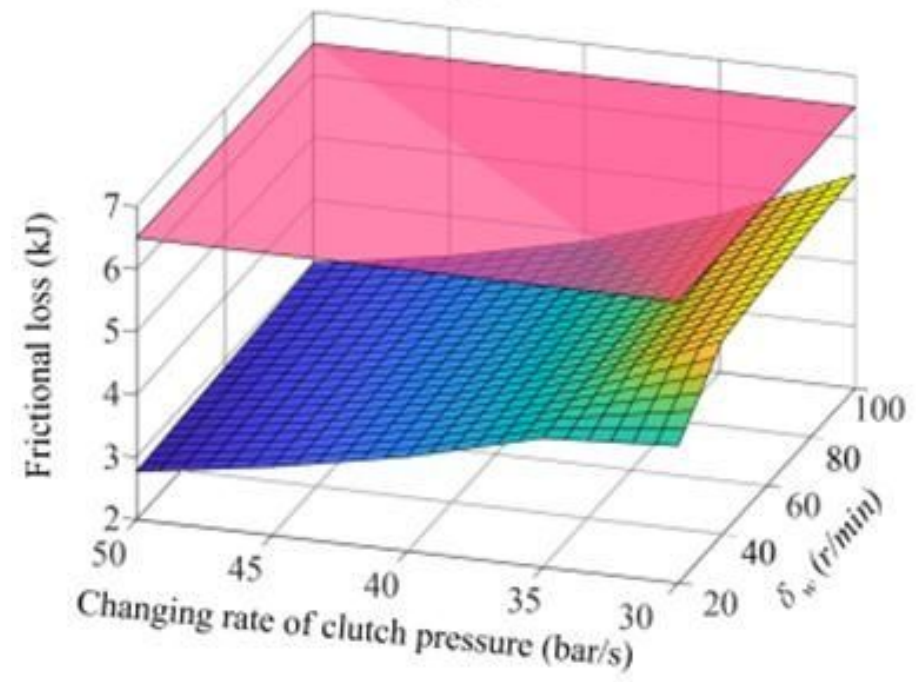

(b)

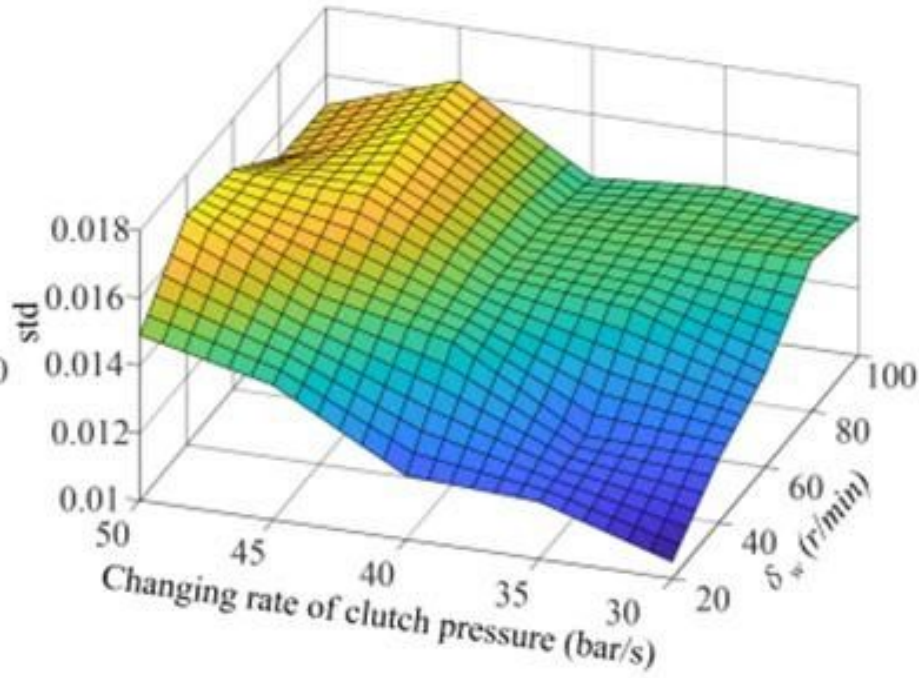

(d)

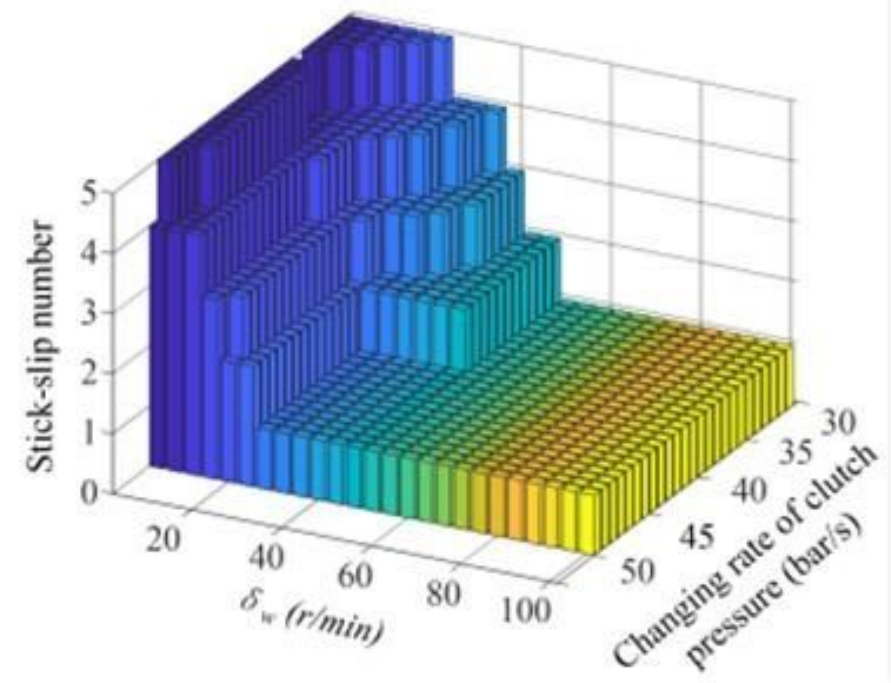

Figure 20

Coupling effect of controllable parameters on dynamic characteristics of driveline at early stage 
(a)

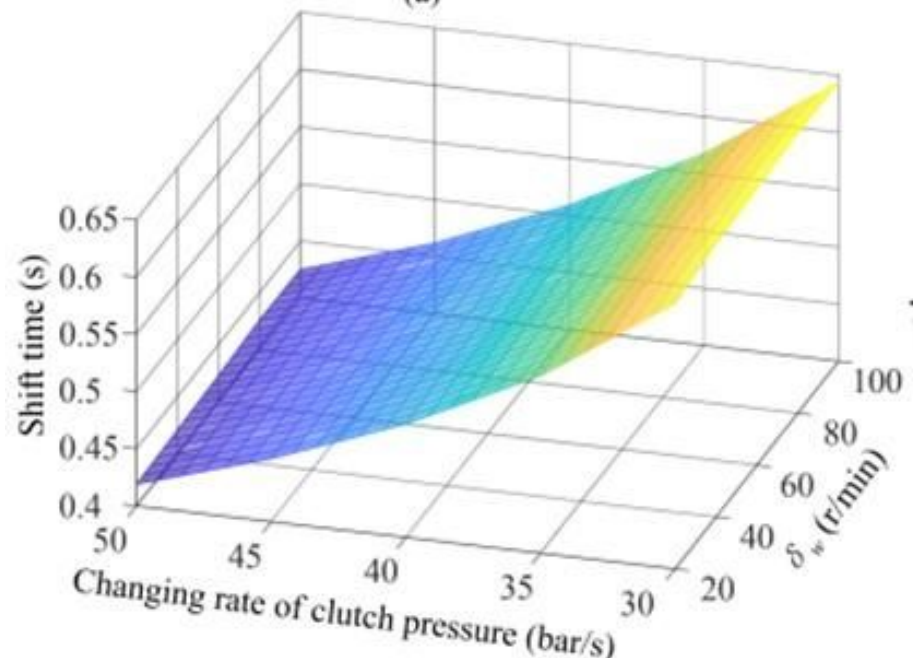

(c)

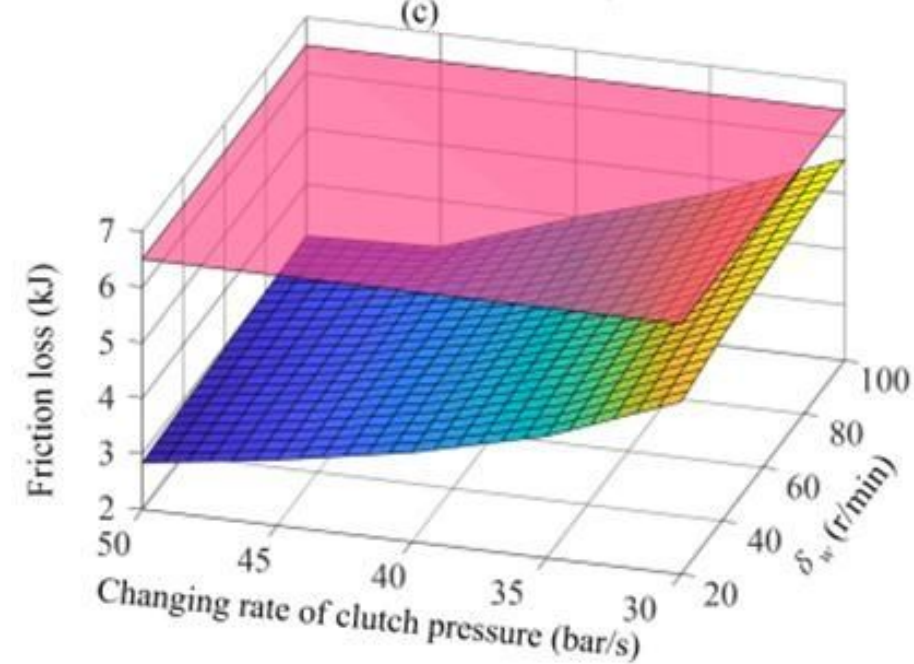

(b)

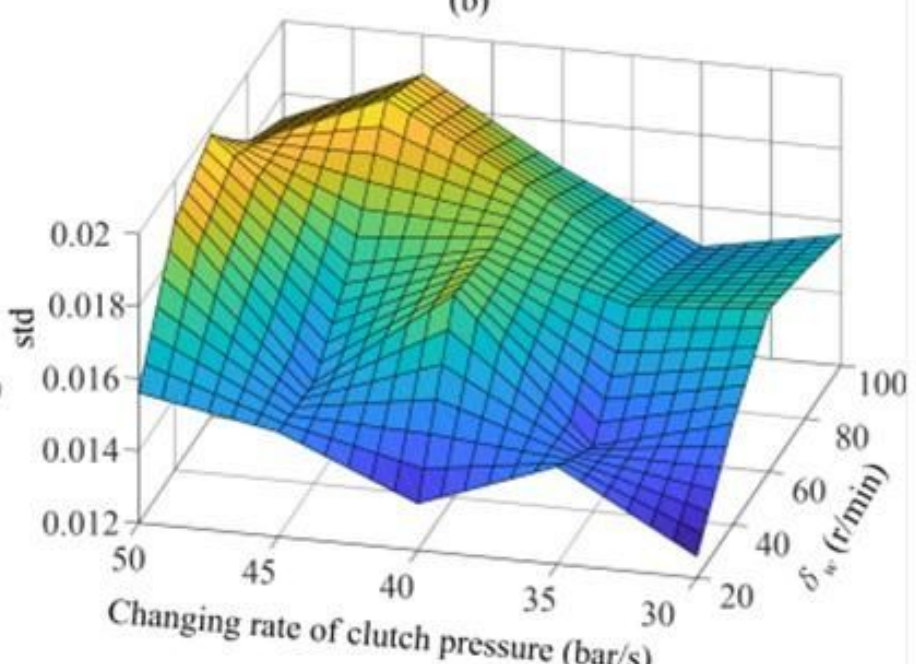

(d)

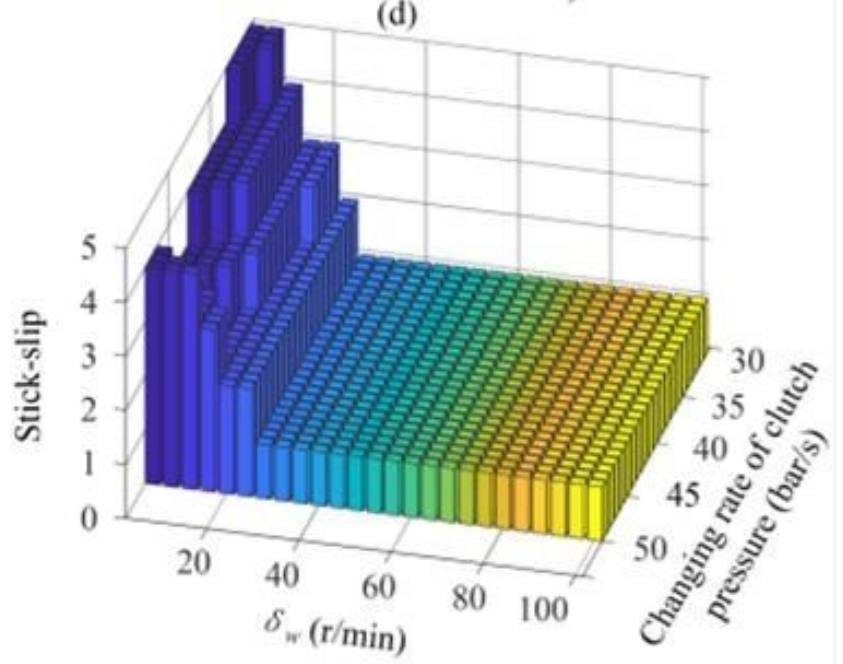

Figure 21

Coupling effect of controllable parameters on dynamic characteristics of driveline at metaphase 
(a)

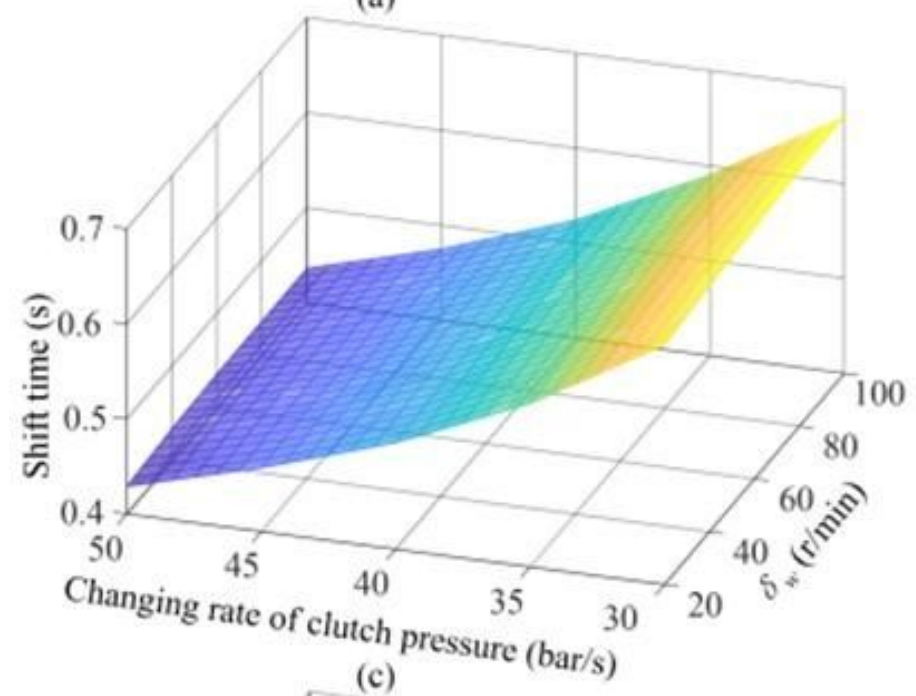

(c)

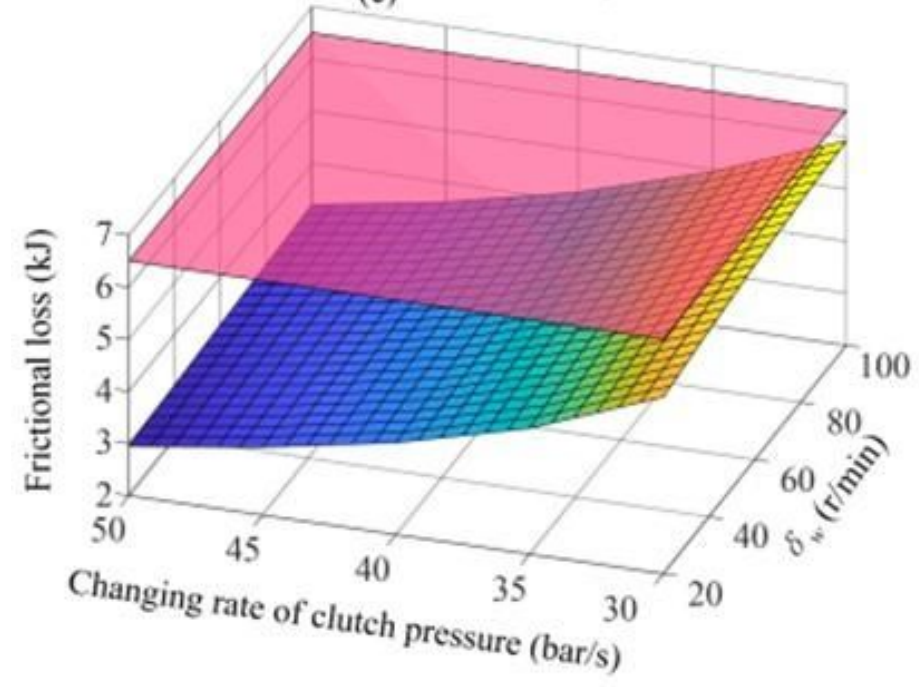

(b)

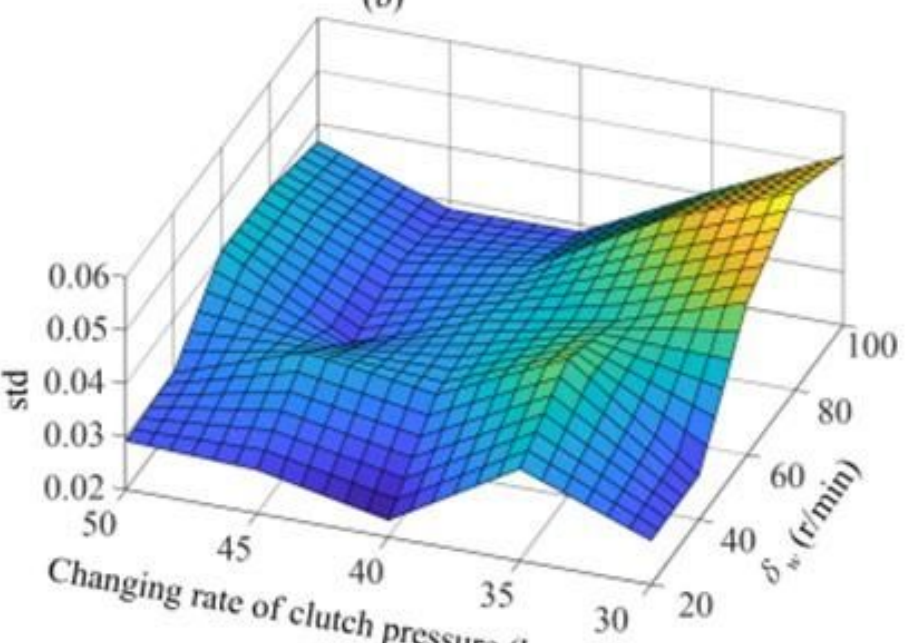

(d)

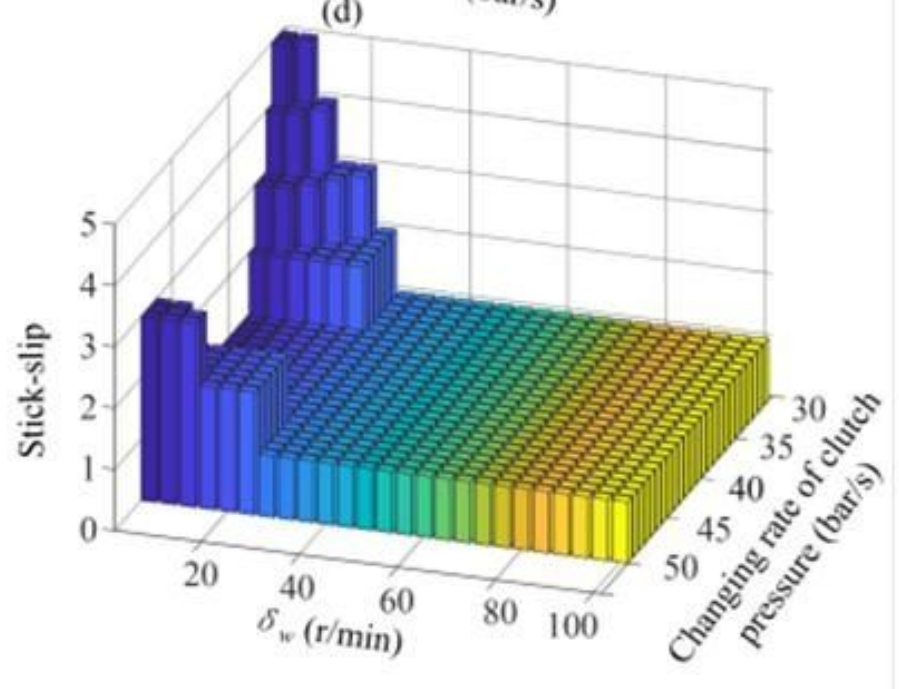

Figure 22

Coupling effects of controllable parameters on dynamic characteristics of driveline at final stage 